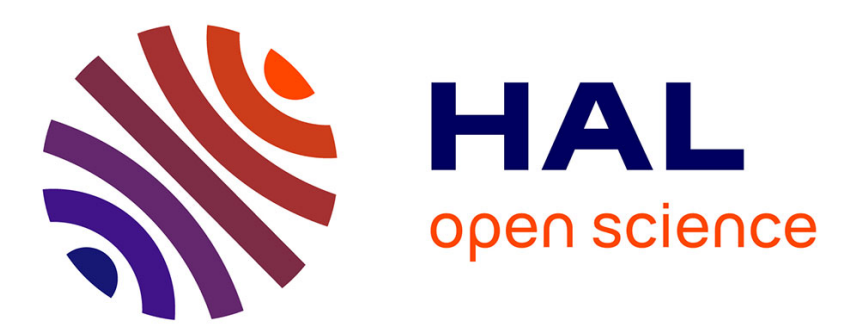

\title{
Formal Verification of Synchronous Data-flow Program Transformations Toward Certified Compilers
}

van Chan Ngo, Loïc Besnard, Paul Le Guernic, Jean-Pierre Talpin, Thierry

Gautier

\section{- To cite this version:}

van Chan Ngo, Loïc Besnard, Paul Le Guernic, Jean-Pierre Talpin, Thierry Gautier. Formal Verification of Synchronous Data-flow Program Transformations Toward Certified Compilers. Frontiers of Computer Science, 2013. hal-00846279

\section{HAL Id: hal-00846279 \\ https://hal.inria.fr/hal-00846279}

Submitted on 18 Jul 2013

HAL is a multi-disciplinary open access archive for the deposit and dissemination of scientific research documents, whether they are published or not. The documents may come from teaching and research institutions in France or abroad, or from public or private research centers.
L'archive ouverte pluridisciplinaire HAL, est destinée au dépôt et à la diffusion de documents scientifiques de niveau recherche, publiés ou non, émanant des établissements d'enseignement et de recherche français ou étrangers, des laboratoires publics ou privés. 


\title{
RESEARCH ARTICLE
}

\section{Formal Verification of Synchronous Data-flow Program Transformations Toward Certified Compilers}

\author{
Van Chan Ngo $(\bowtie)^{1}$, Jean-Pièrre Talpin ${ }^{1}$, Thierry Gautier ${ }^{1}$, \\ Paul Le Guernic ${ }^{1}$, and Loïc Besnard ${ }^{2}$ \\ 1 INRIA Rennes-Bretagne Atlantique, Rennes 35042, France \\ 2 IRISA/CNRS, Rennes 35042, France
}

(c) Higher Education Press and Springer-Verlag Berlin Heidelberg 2012

\begin{abstract}
Translation validation was introduced in the 90's by Pnueli et al. as a technique to formally verify the correctness of code generators. Rather than certifying the code generator or exhaustively qualifying it, translation validators attempt to verify that program transformations preserve semantics. In this work, we adopt this approach to formally verify that the clock semantics and data dependence are preserved during the compilation of the Signal compiler. Translation validation is implemented for every compilation phase from the initial phase until the latest phase where the executable code is generated, by proving that the transformation in each phase of the compiler preserves the semantics.

We represent the clock semantics, the data dependence of a program and its transformed counterpart as first-order formulas which are called Clock Models and Synchronous Dependence Graphs (SDGs), respectively. Then we introduce clock refinement and dependence refinement relations which express the preservation of clock semantics and dependence, as a relation on clock models and SDGs, respectively. Our validator does not require any instrumentation or modification of the compiler, nor any rewriting of the source program.
\end{abstract}

Keywords Formal Verification, Translation Validation, Certified Compiler, Multi-clocked Synchronous Programs, Embedded Systems.

Received May 28, 2013; accepted month dd, yyyy

E-mail: chan.ngo@inria.fr

\section{Introduction}

The synchronous languages such as Esterel [7], Lustre [23] and Signal [16] have been introduced and successfully used to design and implement embedded and critical real-time systems. They have associated compilers, which transform, optimize, and generate code in some general-purpose programming language. Their compilation involves many analyzes, and program transformations. Some transformations may introduce additional information or constraints, to refine the meaning, and/or specialize the behavior of the original program, such as optimization or static scheduling. Thus, the complexity of these compilers increases the risk that their large-scale use may yield bugs. In a safety-critical framework, it is naturally required that the compiler must be formally verified as well to ensure that the source program semantics is preserved.

To circumvent compiler bugs, one can entirely rewrite the compiler with a theorem proving tool such as Coq [13], or check that it is compliant to DO-178C documents [36]. However, these solutions yield a situation where any change of the compiler (e.g. further optimization and update) means redoing the proof. Another approach, which provides ideal separation between the tool under verification and its checker, is trying to verify that the output and the input have the same semantics. In this aim, translation validation was introduced in the 90's by Pnueli et al. [33,34], as a technique to formally verify correctness of code generators. Translation validators can be used to ensure that program transformations do not 
introduce semantic discrepancies, or to help debugging the compiler implementation. Some other works have adopted the translation validation approach in verification of transformations, and optimizations. In [12,28], the programs before and after the transformations and optimizations of a $\mathrm{C}$ compiler are represented in a common intermediate form, then the preservation of semantics is checked by using symbolic execution and the Coq proof assistant.

A compiler generally involves several phases during its compilation process. For instance, the Signal compiler, in its first two phases: calculates the clock information, makes Boolean abstraction, and makes static scheduling. The final phase is the executable code generation. One can try to prove globally that the input program and its final transformed program have the same semantics. However, we believe that a better approach consists in separating the concerns and proving for each phase the preservation of different kinds of semantic properties. In this case of the Signal compiler, the preservation of the semantics can be decomposed into the preservation of clock semantics, data dependence, and value-equivalence of variables. As first contribution to this work, this paper focuses on proving the preservation of clock semantics in the first phases of the Signal compiler. The preservation of clock semantics described in the present contribution will be used to verify the value-equivalence between data-flows in the source program and its generated code. Thanks to clock semantics preservation, the evaluation of a normalizing value-graph [39], used for that purpose, will be more efficient and faster. Moreover, the encoding of clock information considered here will be reused in order to represent the dependence graph of the synchronous programs for studying the preservation of data dependencies which is considered as the other contribution of this work.

The clock semantics of the source program and its transformed counterpart are formally represented as clock models. A clock model is a first-order logic formula with uninterpreted functions. This formula deterministically characterizes the presence/absence status of all discrete data-flows (input, output and local variables of the program) manipulated by the specification at a given instant. Given two clock models, a correct transformation relation between them is defined, which expresses the semantic preservation of clock information. In the implementation, we apply our translation validation to the first two transformation steps of the compiler.

With the similar approach, the dependence in the source program and its transformed counterpart is represented by the formal structure, called Synchronous Dependence Graph
(SDG). A SDG for a given program is a labelled directed graph in which each node is a signal or a clock and each edge represents a dependence between nodes. Each edge is labeled by a clock expression called clock constraint at which the dependence between two extremity nodes is effective. Given two SDGs, a correct transformation relation between them is defined which expresses the semantic preservation of data dependence. In implementation, a SMT-solver is used for checking the existence of the correct transformation relations. We apply this validation to the second transformation steps of the compiler, static scheduling.

At a high level, our tool works as follows. For each transformation, it takes the input program and its transformed counterpart, and constructs the corresponding clock models, SDGs. Then it delegates the existence checking of the correct transformation relation to a solver. If the result is that the relation does not exist then a "compiler bug" message is emitted. Otherwise, the compiler continues its work.

The remainder of this paper is organized as follows. Section 2 introduces the Signal language. Section 3 presents the abstraction that represents the clock constraints in terms of first-order logic formulas. The definition and properties of SDGs are detailed in Section 4. In Section 5, we consider the definitions of correct transformation on clocks and SDGs which formally prove the conformance between the original specification and its compiled counterpart w.r.t the clock semantic and the data dependence. It also addresses the application of the verification process to the Signal compiler, and its integration into the Polychrony toolset [32]. Section 6 presents some related works, concludes our work and outlines future directions.

\section{The Signal Language}

Signal $[9,21]$ is a polychronous data-flow language that allows the specification of multi-clocked systems. Signal handles unbounded series of typed values $(x(t))_{t \in \mathbb{N}}$, called signals, denoted as $x$. Each signal is implicitly indexed by a logical clock indicating the set of instants at which the signal is present, noted $C_{x}$. At a given instant, a signal may be present where it holds a value, or absent (denoted by \#).

\subsection{Language Features}

Syntax. In Signal, a process (written $P$ or $Q$ ) consists of the synchronous composition (noted $P \mid Q$ ) of equations over signals $x, y, z$, written $x:=y f z$ or $x:=f(y, z)$. The process $P / x$ restricts the lexical scope of the signal $x$ to the process 
$P$. An equation $x:=y f z$ defines the output signal $x$ by the result of the application of operator $f$ to its inputs $y, z$.

$$
P, Q::=x:=y f z|P| Q \mid P / x
$$

Semantic Domains. For a set of values (a type) $\mathbb{D}$ we define its extended set $\mathbb{D}_{\#}=\mathbb{D} \cup\{\#\}$, where \# $\notin \mathbb{D}$ is a special symbol used to denote the absence of an occurrence in the signal. $\mathbb{D}_{\#}$ is flat. We denote by $\mathbb{D}^{\infty}=\mathbb{D}^{*} \cup \mathbb{D}^{\omega}$ the set of finite and

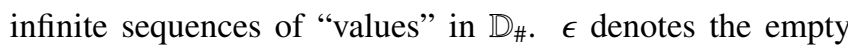
sequence. All signal functions $f: \mathbb{D}_{1}^{\infty} \times \ldots \times \mathbb{D}_{n}^{\infty} \rightarrow \mathbb{D}_{n+1}^{\infty}$ are defined using the following conventions: $x, y, z \ldots$ are signals, $v_{1}, \ldots, v_{n}$ are values in $\mathbb{D}_{i}$ (cannot be $\left.\#\right), v_{1}^{\#}, \ldots, v_{n}^{\#}$ are values in $\mathbb{D}_{i_{\sharp}}$, and $x . y$ is the concatenation of two sequences $x$ and $y$. Signal functions are total, strict and continuous functions over domains [3] (w.r.t prefix order) that satisfy the following general rules:

- $f\left(\# . x_{1}, \ldots, \# . x_{n}\right)=\# . f\left(x_{1}, \ldots, x_{n}\right)$

- $f\left(x_{1}, \ldots, x_{n}\right)=\epsilon$ when for some $i, x_{i}=\epsilon$

A function is synchronous iff it satisfies:

- $f\left(v_{1}^{\#} \cdot x_{1}, \ldots, v_{n}^{\#} \cdot x_{n}\right)=\epsilon$ when $v_{i}^{\#}=\#$ and $v_{j}^{\#} \neq \#$ for some $i, j$

Stepwise Extension $\left(y:=f\left(x_{1}, \ldots, x_{n}\right)\right)$. Given $n>0$ and a n-ary total function $f: \mathbb{D}_{1} \times \ldots \times \mathbb{D}_{n} \rightarrow \mathbb{D}_{n+1}$, the stepwise extension of $f$ denoted $F$ is the synchronous function that satisfies:

- $F\left(v_{1} . x_{1}, \ldots, v_{n} \cdot x_{n}\right)=f\left(v_{1}, \ldots, v_{n}\right) \cdot F\left(x_{1}, \ldots, x_{n}\right)$

Previous Value $(y:=x \$ 1$ init $a)$. The $\$: \mathbb{D}_{i} \times \mathbb{D}_{i}^{\infty} \rightarrow \mathbb{D}_{i}^{\infty}$ is the synchronous (state) function that satisfies

- $\$\left(v_{-1}, v \cdot x\right)=v_{-1} . \$(v, x)$

Deterministic Merge $(y:=x$ default $z$ ). The default: $\mathbb{D}_{i \#}^{\infty} \times \mathbb{D}_{i \#}^{\infty} \rightarrow \mathbb{D}_{i \#}^{\infty}$ signal function is recursively defined by

- for $v \in \mathbb{D}_{i}$, default $\left(v \cdot x, v^{\#} \cdot z\right)=v \cdot \operatorname{default}(x, z)$.

- $\operatorname{default}\left(\# \cdot x, v^{\#} \cdot z\right)=v^{\#} \cdot \operatorname{default}(x, z)$.

Boolean Sampling $(y:=x$ when $b)$. The when: $\mathbb{D}_{i \#}^{\infty} \times \mathbb{B}_{\#}^{\infty} \rightarrow$ $\mathbb{D}_{i \#}^{\infty}$ signal function is recursively defined by

- for $b^{\#} \in \mathbb{B}_{\#}, b^{\#} \neq \operatorname{true}$ when $\left(v^{\#} . x, b^{\#} . b\right)=\#$.when $(x, b)$

- when $\left(v^{\#} \cdot x\right.$, true. $\left.b\right)=v^{\#}$.when $(x, b)$

A network of strict, continuous signal functions that satisfies the Kahn conditions is a strict, continuous signal function or Kahn Process Network [25].

Clock Relations. In addition, the language allows clock constraints to be defined explicitly by some derived operators that can be replaced by primitive operators above. For instance, to define the clock of a signal (represented as an event type signal), $y:=\hat{x}$ specifies that $y$ is the clock of $x$; it is equivalent to $y:=(x=x)$ in the core language. The synchronization $x=y$ means that $x$ and $y$ have the same clock, it can be replaced by $\hat{x}=\hat{y}$. The clock extraction from a Boolean signal is denoted by a unary when: when $b$, that is a shortcut for $b$ when $b$. The clock union $x \hat{+} y$ defines a clock as the union $C_{x} \cup C_{y}$, which can be rewritten as $\hat{x}$ default $\hat{y}$. In the same way, the clock intersection $x * y$ and the clock difference $x{ }^{\wedge}-y$ define clocks $C_{x} \cap C_{y}$ and $C_{x} \backslash C_{y}$, which can be rewritten as $\hat{x}$ when $\hat{y}$ and when $(\operatorname{not}(\hat{y}) \operatorname{default} \hat{x})$, respectively.

Example. The following Signal program emits a sequence of values $F B, F B-1, \ldots, 2,1$, from each value of a positive integer signal $F B$ coming from its environment:

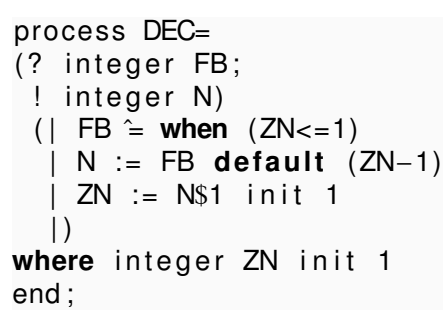

Let us comment this program: ? integer $F B$; ! integer $N$ : $F B, N$ are respectively input and output signals of type integer; $F B^{\wedge}=$ when $(Z N<=1)$ : $F B$ is accepted (or it is present) only when $Z N$ becomes less than or equal to 1 ; $N:=F B$ default $(Z N-1): N$ is set to $F B$ when its previous value is less than or equal to 1 , otherwise it is decremented by $1 ; Z N:=N \$ 1$ init 1 : defines $Z N$ as always carrying the previous value of $N$ (the initial value of $Z N$ is 1); where integer $Z N$ init 1: indicates that $Z N$ is a local signal whose initial value is 1 . Note that the clock of the output signal is more frequent than that of the input. This is illustrated in the following possible trace:

\begin{tabular}{|c|c|c|c|c|c|c|c|c|}
\hline $\mathrm{t}$. & & & . & . & . & . & . & . \\
\hline FB 6 & \# & \# & \# & \# & \# & 3 & \# & \# \\
\hline ZN 1 & 6 & 5 & 4 & 3 & 2 & 1 & 3 & 2 \\
\hline N 6 & 5 & 4 & 3 & 2 & 1 & 3 & 2 & 1 \\
\hline$c_{\mathrm{FB}} t_{\theta}$ & & & & & & $t_{6}$ & & \\
\hline$c_{\mathrm{ZN}} t_{0}$ & $t_{1}$ & $t_{2}$ & $t_{3}$ & $t_{4}$ & $t_{5}$ & $t_{6}$ & $t_{7}$ & $t_{8}$ \\
\hline$c_{N} \quad t_{0}$ & $t_{1}$ & $t_{2}$ & $t_{3}$ & $t_{4}$ & $t_{5}$ & $t_{6}$ & $t_{7}$ & $t_{8}$ \\
\hline
\end{tabular}

Program Structure. The language is modular. In particular, a process can be used as a basic pattern, by means of an interface that describes its parameters and its input and output signals. Moreover, a process can use other subprocesses, or even external parameter processes that are only known by their interfaces. For example, to emit three sequences of values $\left(F B_{i}\right)-1, \ldots, 2,1$ for all three positive integer inputs $F B_{i}$, with $i=1,2,3$, one can define the following process (in 
which, without additional synchronizations, the three subprocesses have unrelated clocks):

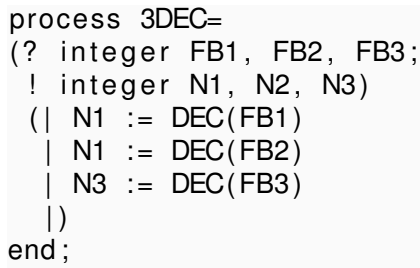

\subsection{Clock Constraints and Dependence}

The above basic processes induce implicitly the clock constraints and dependence between the signals. Table 1 shows these clock constraints for the primitive operators. In this table, the sub-clock $[c]$ (resp. $[\neg c])$ is defined as $\left\{t \in C_{c} \mid c(t)=\right.$ true $\}$ (resp. $\left\{t \in C_{c} \mid c(t)=\right.$ false $\}$ ). Notice that a clock can be viewed as a signal with type event (which has only one value, true, when it is present), thus the condition $C_{c}$ means that the signal $c$ is present.

Let $x, y$ be two signals and $c$ an event or Boolean signal, if at any instant $t$ such that $t \in C_{x} \cap C_{y} \cap C_{c}$ and $c(t)=$ true, setting a value to $y$ cannot precede the availability of $x$, then we say that $y$ depends on $x$ at the condition $c$. We use $x \stackrel{c}{\rightarrow} y$ to denote the fact that there is a dependence between $y$ and $x$ at the condition $c$. Table 1 shows the dependence for the core language. In particular, the following dependence applies equally: i) Any signal is preceded by its clock. ii) For a Boolean signal $c,[c]$ and $[\neg c]$ depend on $c$. iii) Any dependence $x \stackrel{c}{\rightarrow} y$ implies implicitly a dependence $[c] \stackrel{C_{c}}{\rightarrow} y$. As an example, for the basic process corresponding to the primitive operator Boolean sampling, the clock constraints and dependence between signals are given by:

- The clock of $y$ is the intersection of the clock of $x$ and the sub-clock $[b]$.

- The signal $y$ depends on the signal $x$ whenever $y$ is present.

- The clock $C_{y}$ depends on the Boolean signal $b$ whenever $y$ is present.

\subsection{Compilation of Signal Programs}

The Signal compiler [8] consists of a sequence of code transformations. Some transformations are optimizations that rewrite the code to eliminate inefficient expressions. The compilation process may be seen as a sequence of morphisms rewriting Signal programs to Signal programs. The final steps (C or Java code generation) are simple morphisms over the ultimately transformed program. For convenience, the transfor-

\begin{tabular}{|c|c|c|}
\hline & Dependence & Clock Constraint \\
\hline$x$ & $C_{x} \stackrel{C_{x}}{\longrightarrow} x$ & \\
\hline$c$ (Boolean signal) & $\begin{array}{l}c \stackrel{C_{c}}{\longrightarrow}[c] \\
c \stackrel{C_{c}}{\longrightarrow}[\neg c]\end{array}$ & \\
\hline$x \stackrel{c}{\rightarrow} y$ & {$[c] \stackrel{C_{c}}{\longrightarrow} y$} & \\
\hline$y:=f\left(x_{1}, \ldots, x_{n}\right)$ & $\begin{array}{l}x_{1} \stackrel{C_{y}}{\longrightarrow} y \\
\cdots \\
x_{n} \stackrel{C_{y}}{\longrightarrow} y\end{array}$ & $\begin{array}{l}C_{y}=C_{x_{1}} \\
\ldots \\
C_{y}=C_{x_{n}}\end{array}$ \\
\hline$y:=x \$ 1$ init $a$ & $y \stackrel{C_{y}}{\longrightarrow} x$ & $C_{y}=C_{x}$ \\
\hline$y:=x$ when $b$ & $\begin{array}{l}x \stackrel{C_{y}}{\longrightarrow} y \\
b \stackrel{C_{y}}{\longrightarrow} C_{y}\end{array}$ & $C_{y}=C_{x} \cap[b]$ \\
\hline$y:=x$ default $z$ & $\begin{array}{l}x \stackrel{C_{x}}{\longrightarrow} y \\
z \stackrel{C_{z} \backslash C_{x}}{\longrightarrow} y\end{array}$ & $C_{y}=C_{x} \cup C_{z}$ \\
\hline
\end{tabular}

Table 1 The Clock Constraints and Dependence

mations of the compiler are divided into three phases as depicted in Figure 1. The optimized final program *_SEQ_TRA is translated directly to executable code. Signal programs

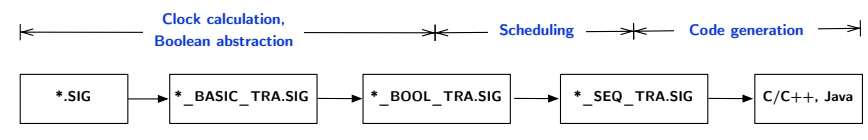

Fig. 1 The compilation of Signal compiler

which are produced in the first two phases (clock calculation, Boolean abstraction and static scheduling) have the following features:

- The transformed programs are also written in Signal language.

- The clocks of all signals have been calculated and the overall set of clocks is organized as a clock hierarchy which is a set of clock trees [8]. When there is a single clock tree, the process has a fastest rated clock and it is said endochronous. When there are several clock trees, the process may be endochronized with an explicit parameterization, adding a fastest clock, Tick.

- In the successive transformations of the compiler, clocks are first represented as event signals related through clock specific Signal operators (this is reflected in the *_BASIC_TRA intermediate form); then clocks are transformed into Boolean signals defined with Boolean operators (this is reflected in the *_BOOL_TRA intermediate form).

- The scheduling information is represented in the 
*_SEQ_TRA intermediate form.

- The arithmetic expressions are leaved intact.

As an example, the body of the intermediate form DEC_BASIC_TRA obtained by compiling the above DEC process is as follows:

(| CLK := CLK N $\wedge_{-}$CLK FB $\left.\mid\right)$

(| $\operatorname{CLKN}:=\operatorname{CLKN}{ }^{\wedge}+$ CLK FB

CLK $\mathrm{N}^{\wedge}=\mathrm{N}^{\wedge}=\mathrm{ZN}$

| $(\mid \bar{N}:=$ (FB when CLK_FB)

default $((\mathrm{ZN}-1)$ when $\mathrm{CLK})$

| $Z N:=N \$ 1$ init 1

1)

| ( $\mathrm{CLK}$ FB $:=$ when $(\mathrm{ZN}<=1)$

CLK FB ${ }^{\wedge}=$ FB

CLK_12 := when $(\operatorname{not}(\mathrm{ZN}<=1))$

1)

l)

\section{Clock Models}

In this section, we describe the timing semantics of a program in terms of a first-order logic formula. Let us consider the semantics of the sampling operator $y:=x$ when $b$. At any instant, the signal $y$ holds the value of $x$ if the following conditions are satisfied: $x$ holds a value, and $b$ is present and holds the value true; otherwise, it holds no value. Thus, to represent the underlying control conditions, we need to model the statuses present with value true or false and absent for the signal $b$, and the statuses present and absent for the signal $x$. This section explores a method to construct the control model of a program as an abstraction of the clock semantics, called clock model, which is the computational model of our translation validation approach.

\subsection{Illustrative Example}

In Signal, clocks play a much more important role than in other synchronous languages, they are used to express the underlying control (i.e., the synchronization between signals) for any conditional definition. This differs from Lustre, where all clocks are built by sampling the fastest clock. For instance, we consider again the basic process corresponding to the primitive operator Boolean sampling, where $x$ and $y$ are numerical signals, and $b$ is a Boolean signal: $y:=x$ when $b$. To express the control, we need to represent the status of the signals $x, y$ and $b$ at a given instant. In this example, we use a Boolean variable $\hat{x}$ to capture the status of $x$ : $(\hat{x}=$ true $)$ means $x$ is present, and ( $\hat{x}=$ false) means $x$ is absent. In the same way, the Boolean variable $\hat{y}$ captures the status of $y$. For the Boolean signal $b$, two Boolean variables $\hat{b}$ and $\bar{b}$ are used to represent its status: $(\hat{b}=$ true $\wedge \bar{b}=$ true $)$ means $b$ is present and holds a value true; $(\hat{b}=$ true $\wedge \bar{b}=$ false) means $b$ is present and holds a value false; and ( $\hat{b}=$ false $)$ means $b$ is absent.

Hence, at a given instant, the implicit control relations of the basic process above can be encoded by the following formula:

$$
\hat{y} \Leftrightarrow(\hat{x} \wedge \hat{b} \wedge \bar{b})
$$

\subsection{Abstraction}

Let $X=\left\{x_{1}, \ldots, x_{n}\right\}$ be the set of all signals in program $P$. With each signal $x_{i}$, we attach a Boolean variable $\widehat{x_{i}}$ to encode its clock and a variable $\overline{x_{i}}$ of same type as $x_{i}$ to encode its value. Formally, the abstract values which represent the clock semantics of the program can be computed using the following functions:

^ $X \longrightarrow \mathbb{B}$ associates a signal with a Boolean value;

${ }^{-}: X \longrightarrow \mathbb{D}$ associates a signal with a value of same type as the signal.

The composition of Signal processes corresponds to logical conjunctions. Thus clock model of $P$ will be a conjunction $\Phi(P)=\bigwedge_{i=1}^{n} \phi\left(e q_{i}\right)$ whose atoms are $\widehat{x_{i}}, \overline{x_{i}}$, where $\phi\left(e q_{i}\right)$ is the abstraction of statement $e q_{i}$ (statement using the Signal primitive operators), and $n$ is the number of statements in the program. In the following, we present the abstraction corresponding to each Signal operator.

\subsubsection{Stepwise Extension}

The functions which apply on signal values in the primitive stepwise functions are usual logic operators (not, and, or), numerical comparison functions $(<,>,=,<=,>=, /=)$, and numerical operators $(+,-, *, /)$. In our experience working with the Signal compiler, it performs very few arithmetical optimizations and leaves most of the arithmetical expressions intact. Every variable is determinable by the inputs, memorizable values, otherwise program can not be compiled. This suggests that most of the implications will hold independently of the features of the numerical comparison functions and numerical operators and we can replace the operations by uninterpreted functions. By following the encoding procedure of [1], for every numerical comparison functions and numerical operator (denoted by $\square$ ) occurring in an equation, we perform the following rewriting:

- Replace each $x \square y$ by a new variable $v_{\square}^{i}$ of a type equal to that of the value returned by $\square$. Two stepwise functions $x \square y$ and $x^{\prime} \square y^{\prime}$ are replaced by the same variable $v_{\square}^{i}$ iff $x, y$ are identical to $x^{\prime}$ and $y^{\prime}$, respectively. 
- For every pair of newly added variables $v_{\square}^{i}$ and $v_{\square}^{j}, i \neq j$, corresponding to the non-identical occurrences $x \square y$ and $x^{\prime} \square y^{\prime}$, add the implication $\left(\bar{x}=\overline{x^{\prime}} \wedge \bar{y}=\overline{y^{\prime}}\right) \Rightarrow$ $\overline{v_{\square}^{i}}=\overline{v_{\square}^{j}}$ into the abstraction $\Phi(P)$.

The abstraction $\phi\left(y:=f\left(x_{1}, \ldots, x_{n}\right)\right)$ of stepwise functions is defined by induction as follows:

- $\phi($ true $)=$ true and $\phi($ false $)=$ false

- $\phi(y:=x)=(\hat{y} \Leftrightarrow \hat{x}) \wedge(\hat{y} \Rightarrow(\bar{y} \Leftrightarrow \bar{x}))$ if $x$ and $y$ are Boolean. $\phi(y:=x)=(\hat{y} \Leftrightarrow \hat{x}) \wedge(\hat{y} \Rightarrow(\bar{y} \Leftrightarrow \bar{x})) \wedge(\hat{x} \Rightarrow \bar{x})$ if $x$ is an event signal.

- $\phi\left(y:=x_{1}\right.$ and $\left.x_{2}\right)=\left(\hat{y} \Leftrightarrow \widehat{x_{1}} \Leftrightarrow \widehat{x_{2}}\right) \wedge(\hat{y} \Rightarrow(\bar{y} \Leftrightarrow$ $\left.\left.\overline{x_{1}} \wedge \overline{x_{2}}\right)\right)$.

- $\phi\left(y:=x_{1}\right.$ or $\left.x_{2}\right)=\left(\hat{y} \Leftrightarrow \widehat{x_{1}} \Leftrightarrow \widehat{x_{2}}\right) \wedge\left(\hat{y} \Rightarrow\left(\bar{y} \Leftrightarrow \overline{x_{1}} \vee \overline{x_{2}}\right)\right)$.

- $\left.\underline{\phi(y}:=x_{1} \square x_{2}\right)=\left(\hat{y} \Leftrightarrow \widehat{v_{\square}^{i}} \Leftrightarrow \widehat{x_{1}} \Leftrightarrow \widehat{x_{2}}\right) \wedge(\hat{y} \Rightarrow(\bar{y}=$ $\left.\left.\overline{v_{\square}^{i}}\right)\right)$.

\subsubsection{Previous Value}

Considering the previous value operator, $y:=x \$ 1$ init $a$, its encoding $\phi(y:=x \$ 1$ init $a)$ contributes to $\Phi(P)$ with the following conjunct:

- if $x, y$ and $a$ are Boolean:

$(\hat{y} \Leftrightarrow \hat{x})$

$\wedge\left(\hat{y} \Rightarrow\left((\bar{y} \Leftrightarrow m \cdot x) \wedge\left(m \cdot x^{\prime} \Leftrightarrow \bar{x}\right)\right)\right)$

$\wedge\left(m \cdot x_{0} \Leftrightarrow a\right)$

- if $x, y$ and $a$ are non-Boolean:

$(\hat{y} \Leftrightarrow \hat{x})$

This encoding requires that at any instant, signals $x$ and $y$ have the same status (present or absent). If the signals are Boolean, it encodes the value of the output signal as well. Here, we introduce a memorization variable $m . x$ that stores the last value of $x$. The next value of $m . x$ is $m \cdot x^{\prime}$ and it is initialized to $a$ in $m \cdot x_{0}$.

\subsubsection{Deterministic Merge}

The encoding of the deterministic merge operator, $y:=$ $x$ default $z$, contributes to $\Phi(P)$ with the following conjunct:

- if $x, y$ and $z$ are Boolean:

$(\hat{y} \Leftrightarrow(\hat{x} \vee \hat{z}))$

$\wedge \hat{y} \Rightarrow((\hat{x} \wedge(\bar{y} \Leftrightarrow \bar{x}))$

$\vee(\neg \hat{x} \wedge(\bar{y} \Leftrightarrow \bar{z}))))$

- if $x, y$ and $z$ are non-Boolean: $\hat{y} \Leftrightarrow(\hat{x} \vee \hat{z})$

\subsubsection{Boolean Sampling}

The encoding of the Boolean sampling operator, $y:=$ $x$ when $b$, contributes to $\Phi(P)$ with the following conjunct:

- if $x$ and $y$ are Boolean:

$(\hat{y} \Leftrightarrow(\hat{x} \wedge \hat{b} \wedge \bar{b}))$

$\wedge(\hat{y} \Rightarrow(\bar{y} \Leftrightarrow \bar{x}))$

- if $x$ and $y$ are non-Boolean: $\hat{y} \Leftrightarrow(\hat{x} \wedge \hat{b} \wedge \bar{b})$

\subsubsection{Composition}

Consider the composition of two processes $P_{1}$ and $P_{2}$. Its abstraction $\phi\left(P_{1} \mid P_{2}\right)$ is defined as follows:

- $\phi\left(P_{1}\right) \wedge \phi\left(P_{2}\right)$

\subsubsection{Clock Relations}

Given the above rules, we can obtain the following abstraction for derived operators on clocks. Here, $z$ is a signal of type event:

- $\phi(z:=\hat{x})=(\hat{z} \Leftrightarrow \hat{x}) \wedge(\hat{z} \Rightarrow \bar{z})$

- $\phi\left(x^{\wedge}=y\right)=\hat{x} \Leftrightarrow \hat{y}$

- $\phi\left(z:=x^{\wedge}+y\right)=(\hat{z} \Leftrightarrow(\hat{x} \vee \hat{y})) \wedge(\hat{z} \Rightarrow \bar{z})$

- $\phi\left(z:=x^{\wedge} * y\right)=(\hat{z} \Leftrightarrow(\hat{x} \wedge \hat{y})) \wedge(\hat{z} \Rightarrow \bar{z})$

- $\phi\left(z:=x^{\wedge}-y\right)=(\hat{z} \Leftrightarrow(\hat{x} \wedge \neg \hat{y})) \wedge(\hat{z} \Rightarrow \bar{z})$

- $\phi(z:=$ when $b)=(\hat{z} \Leftrightarrow(\hat{b} \wedge \bar{b})) \wedge(\hat{z} \Rightarrow \bar{z})$

\subsubsection{Nested processes}

Assume that a process $P$ has a sub-process $P_{1}$, the abstraction $\Phi(P)$ is given by:

- $\phi(P) \wedge \phi\left(P_{1}\right)$

- For every equation in process $P$ that involves an invocation of a sub-process such as $\left(y_{1}, \ldots, y_{n}\right):=P_{1}\left(x_{1}, \ldots, x_{m}\right)$, the following conjuncts are added, where $i_{h}, o_{k}$ are the inputs and outputs of $P_{1}$ :

$$
\bigwedge_{k=1}^{n}\left(\widehat{y_{k}} \Leftrightarrow \widehat{o_{k}} \wedge \overline{y_{k}} \Leftrightarrow \overline{o_{k}}\right) \wedge \bigwedge_{h=1}^{m}\left(\widehat{x_{h}} \Leftrightarrow \widehat{i_{h}} \wedge \overline{x_{h}} \Leftrightarrow \overline{i_{h}}\right)
$$

Applying the abstraction rules above, the clock semantics of the Signal program $D E C$ is represented by the following firstorder logic formula $\Phi(\mathrm{DEC})$, where $Z N<=1$ is replaced by $v_{<=}^{1}$ and $Z N-1$ is replaced by $v_{-}^{1}$.

$$
\begin{aligned}
& \left(\widehat{F B} \Leftrightarrow \widehat{v_{<=}^{1}} \wedge \overline{v_{<=}^{1}}\right) \\
\wedge & \left(\widehat{v_{<=}^{1}} \Leftrightarrow \widehat{Z N}\right) \\
\wedge & (\widehat{Z N} \Leftrightarrow \widehat{N}) \\
\wedge & \left(\widehat{N} \Leftrightarrow \widehat{F B} \vee \widehat{v_{-}^{1}}\right) \\
\wedge & \left(\widehat{v_{-}^{1}} \Leftrightarrow \widehat{Z N}\right)
\end{aligned}
$$




\subsection{Concrete Clock Semantics}

Let $X_{\mathbb{B}} \subseteq X$ be the set of all Boolean or event signals. We rely on the basic elements of trace semantics [20] to define the clock semantics of a synchronous program.

Definition 1. (Clock events) Given a non-empty set X, the set of clock events on $X$, denoted by $\mathcal{E} c_{X}$, is the set of all possible interpretations $I$ for $X$ and $\bar{I}$ for $X_{\mathbb{B}}$. The interpretations $I, \bar{I}$ are respectively mappings from $X^{n}$ to $\mathbb{B}^{n}$ and from $X_{\mathbb{B}}^{m}$ to $\mathbb{B}^{m}$, where $I(x)=$ true if $x$ holds a value while $I(x)=$ false if it holds no value; and $\bar{I}(x)=$ true if $x$ holds the value true, $\bar{I}(x)=$ false, otherwise.

For example, consider a program whose variables are $X=$ $\{x, b\}$ where $b$ is Boolean variable, the set of clock events is $\mathcal{E} c_{X}=\left\{\left(x \mapsto_{I}\right.\right.$ false, $b \mapsto_{I}$ false, $b \mapsto_{\bar{I}}$ false $),\left(x \mapsto_{I}\right.$ fa - lse, $b \mapsto_{I}$ true, $b \mapsto_{\bar{I}}$ false), $\left(x \mapsto_{I}\right.$ false, $b \mapsto_{I}$ true, $b \mapsto_{\bar{I}}$ true), $\left(x \mapsto_{I}\right.$ true, $b \mapsto_{I}$ false, $b \mapsto_{\bar{I}}$ false), $\left(x \mapsto_{I}\right.$ true, $b \mapsto_{I}$ true, $b \mapsto_{\bar{I}}$ false), $\left(x \mapsto_{I}\right.$ true, $b \mapsto_{I}$ true, $b \mapsto_{\bar{I}}$ true) $\}$. Then at a given instant, the signals clock information is one of these clock events. By convention, the set of clock events of the empty set is defined as the empty set $\mathcal{E} c_{\emptyset}=\emptyset$.

Definition 2. (Clock traces) Given a non-empty set X, the set of clock traces on $X$, denoted by $\mathcal{T} c_{X}$, is defined by the set of functions $T_{c}$ defined from the set $\mathbb{N}$ of natural numbers to $\mathcal{E} c_{X}$, denoted by $T_{c}: \mathbb{N} \longrightarrow \mathcal{E} c_{X}$.

The natural numbers represent the instants $t=0,1,2, \ldots$. A trace $T c$ is a chain of clock events. We denote the interpreted value (true or false) of a variable $x_{i}$ at instant $t$ by $T_{c}(t)\left(x_{i}\right)$, and $\overline{T_{c}(t)\left(x_{i}\right)}$ if $x_{i} \in X_{\mathbb{B}}$. Considering the above example, we have $T_{c}:\left(0,\left(x \mapsto_{I}\right.\right.$ false, $b \mapsto_{I}$ false, $b \mapsto_{\bar{I}}$ false $),\left(1,\left(x \mapsto_{I}\right.\right.$ false, $b \mapsto_{I}$ true, $b \mapsto_{\bar{I}}$ false $\left.)\right), \ldots$ as one of the possible clock traces on $X$, and $T_{c}(0)(x)=$ $T_{c}(0)(b)=\overline{T_{c}(0)(b)}=$ false.

Definition 3. (Clock trace restriction) Given a non-empty set $X$, a subset $X_{1} \subseteq X$, and a clock trace $T_{c}$ being defined on $X$, the restriction of $T_{c}$ onto $X_{1}$ is denoted by $X_{1} . T_{c}$. It is defined as $X_{1} \cdot T_{c}: \mathbb{N} \longrightarrow \mathcal{E} c_{X_{1}}$ such that $\forall t \in \mathbb{N}, \forall x \in$ $X_{1}, X_{1} \cdot T_{c}(t)(x)=T_{c}(t)(x)$ and $\overline{X_{1} \cdot T_{c}(t)(x)}=\overline{T_{c}(t)(x)}$ if $x \in$ $X_{\mathbb{B}}$.

We write $[[P]]_{c}$ to denote the clock semantics of program $P$ which is defined as a set of possible clock traces.

Let $\hat{X}=\left\{\widehat{x_{1}}, \ldots, \widehat{x_{n}}, \overline{x_{1}}, \ldots, \overline{x_{n}}\right\} \cup \widehat{V} \cup \bar{V}$ be a finite set of variables that are used to construct the abstraction, where $V$ is a set of newly added variables in uninterpreted functions replacement. Considering an interpretation $\hat{I}$ over $\hat{X}$, it is called a clock configuration iff it is a model of the firstorder logic formula $\Phi(P)$. For example, $(\widehat{F B} \mapsto$ false, $\widehat{N} \mapsto$ true, $\widehat{Z N} \mapsto$ true) is a clock configuration of $\Phi(\mathrm{DEC})$, but ( $\widehat{F B} \mapsto$ false, $\widehat{N} \mapsto$ true, $\widehat{Z N} \mapsto$ false) is not one (we omit to write the interpretation for other variables).

Given a clock configuration $\hat{I}$, the set of clock events according to $\hat{I}$ and the set of all clock events of $\Phi(P)$ are computed as follows:

$$
\begin{array}{r}
S_{\text {sat }}(\hat{I})=\left\{I \in \mathcal{E} c_{X} \mid \forall i, I\left(x_{i}\right)=\hat{I}\left(\widehat{x_{i}}\right)\right. \\
\text { and } \left.\bar{I}\left(x_{i}\right)=\hat{I}\left(\overline{x_{i}}\right) \text { if } x_{i} \in X_{\mathbb{B}}\right\} \\
S_{\text {sat }}(\Phi(P))=\bigcup_{\hat{I} \models \Phi(P)} S_{\text {sat }}(\hat{I})
\end{array}
$$

With a set of clock events $S_{\text {sat }}(\Phi(P))$, the concrete clock semantics of $\Phi(P)$ is defined by the following set of clock traces:

$$
\Gamma(\Phi(P))=\left\{T_{c} \in \mathcal{T} c_{X} \mid \forall t, T_{c}(t) \in S_{s a t}(\Phi(P))\right\}
$$

\subsection{Soundness of the Abstraction}

Table 2 and 3 show the clock semantics of the primitive operators with non-Boolean and Boolean signals, respectively. For instance, the clock semantics of the basic process corresponding to Boolean sampling is the following set of clock traces:

$T_{c}=\left\{\left(0,\left(c_{x_{0}}, c_{b_{0}}, b_{0}, c_{y_{0}}\right)\right), \ldots,\left(i,\left(c_{x_{i}}, c_{b_{i}}, b_{i}, c_{y_{i}}\right)\right), \ldots\right\}$ s.t $\forall i,\left(c_{x_{i}}, c_{b_{i}}, b_{i}, c_{y_{i}}\right) \in\{($ false, false, false, false $)$, (true, false, false, false), (false, true, false, false), (false, true, true, false), (true, true, false, false), (true, true, true, true)\}

Definition 4. Given the abstraction $\Phi(P)$, a property $\varphi$ defined over the set of clocks $\hat{X}$ is satisfied by $\Phi(P)$ if for any interpretation $\hat{I}, \hat{I} \vDash \Phi(P)$ whenever $\hat{I} \vDash \varphi$, denoted by $\Phi(P) \vDash \varphi$.

To show the soundness of our abstraction, we consider a similar reasoning as in [19]. Our abstraction above is sound in terms of preservation of the clock semantics of the abstracted program $P$ : if the clock semantics of the abstraction satisfies a property defined over the clocks, then the abstracted program also satisfies this property as stated by the following proposition. For any property $\varphi$ which is defined over the set $\hat{X}$, its concretization $\Gamma(\varphi)$ is given by:

$$
\begin{array}{r}
S_{\text {sat }}(\varphi)=\bigcup_{\hat{I}=\varphi} S_{\text {sat }}(\hat{I}) \\
\Gamma(\varphi)=\left\{T_{c} \in \mathcal{T} c_{X} \mid \forall t, T_{c}(t) \in S_{\text {sat }}(\varphi)\right\}
\end{array}
$$


Proposition 1. Let $P, \Phi(P)$ be a program and its abstraction, respectively, and $\varphi$ is a property defined over the clocks. If $\Phi(P) \vDash \varphi$ then $[[P]]_{c} \subseteq \Gamma(\varphi)$.

Lemma 1. For all programs $P,[[P]]_{c} \subseteq \Gamma(\Phi(P))$.

Proof. (Proposition 1) The proof of Proposition 1 is done by using Lemma 1. Given a clock trace $T_{c} \in[[P]]_{c}$, applying Lemma $1, T_{c} \in \Gamma(\Phi(P))$ means that $\forall t, T_{c}(t) \in S_{s a t}(\Phi(P))$. Since $\Phi(P) \vDash \varphi$, then every interpretation $\hat{I}$ satisfying $\Phi(P)$ also satisfies $\varphi$. Thus, any clock event $I \in S_{\text {sat }}(\Phi(P))$ is also in $S_{s a t}(\varphi)$, meaning that $\forall t, T_{c}(t) \in S_{s a t}(\varphi)$. Therefore, we have $T_{c} \in \Gamma(\varphi)$.

Proof. (Lemma 1) We prove it by induction on the structure of program $P$, meaning that for every primitive operator of the language we show that its clock semantics is a subset of the corresponding concretization.

- Stepwise Extensions: $P$ : $y:=f\left(x_{1}, \ldots, x_{n}\right)$. First, consider $y$ as numerical signal; following the encoding scheme, we have $\Phi(P)=\left(\hat{y} \Leftrightarrow \widehat{v_{f}^{i}} \Leftrightarrow \widehat{x_{1}} \Leftrightarrow \ldots \Leftrightarrow \widehat{x_{n}}\right)$. For any interpretation $\hat{I}$ such that $\hat{I} \models \Phi(P)$, we have:

- either $\forall i, \hat{y}=0$ and $\widehat{x_{i}}=0$;

- or $\forall i, \hat{y}=1$ and $\widehat{x_{i}}=1$.

$S_{\text {sat }}(\Phi(P))$ is the set of all interpretations of the form above. Let $T_{c} \in[[P]]_{c}$ be a clock trace and $t \in \mathbb{N}$ be any instant, then either $\forall i, T_{c}(t)(y)=T_{c}\left(x_{i}\right)=0$ or $T_{c}(t)(y)=$ $T_{c}\left(x_{i}\right)=1$, thus $T_{c} \in \Gamma(\Phi(P))$. When $y$ is a boolean signal, the proof is similar.

- Previous Value, Boolean Sampling, and Deterministic Merging operators: we prove in the same manner.

- Composition: $P=P 1 \mid P 2$. Let $T_{c} \in[[P]]_{c}$ be a clock trace, since $X_{1} \cdot T_{c} \in\left[\left[P_{1}\right]\right]_{c}, X_{2} \cdot T_{c} \in\left[\left[P_{2}\right]\right]_{c},\left[\left[P_{1}\right]\right] \subseteq$ $\Gamma\left(\Phi\left(P_{1}\right)\right)$ and $\left[\left[P_{2}\right]\right]_{c} \subseteq \Gamma\left(\Phi\left(P_{2}\right)\right)$, we have $\forall t, T_{c}(t) \in$ $S_{\text {sat }}\left(\Phi\left(P_{1}\right)\right)$ and $T_{c}(t) \in S_{\text {sat }}\left(\Phi\left(P_{2}\right)\right)$. That means $\forall t, T_{c}(t) \in S_{s a t}\left(\Phi\left(P_{1}\right) \wedge \Phi\left(P_{2}\right)\right)$, or $T_{c} \in \Gamma(\Phi(P))$.

\section{Synchronous Dependence Graphs}

The SDG represents a synchronous program as a labelled directed graph in which each node is a signal or a clock and each edge from a node to another node represents the dependence between nodes. Each edge is labeled by a clock constraint.

Thus, a dependence between two signals is conditioned: it means that the dependence is effective whenever the condition holds. For instance, $y:=x$ when $b$ specifies that at any

\begin{tabular}{|c|c|}
\hline Process $P$ & Clock Semantics $[[P]]_{c}$ \\
\hline$y:=f\left(x_{1}, \ldots, x_{n}\right)$ & $\begin{array}{c}\left\{T_{c} \in \mathcal{T} c_{\left\{y, x_{1}, \ldots, x_{n}\right\}} \mid \forall t \in \mathbb{N},\right. \\
\left.\left(\forall i, T_{c}(t)\left(x_{i}\right)=T_{c}(t)(y)\right)\right\}\end{array}$ \\
\hline$y:=x \$ 1$ init $a$ & $\begin{array}{c}\left\{T_{c} \in \mathcal{T} c_{\{x, y\}} \mid \forall t \in \mathbb{N}\right. \\
\left.\left(T_{c}(t)(x)=T_{c}(t)(y)\right)\right\}\end{array}$ \\
\hline$y:=x$ when $b$ & $\begin{array}{c}\left\{T_{c} \in \mathcal{T} c_{\{x, y, b\}} \mid \forall t \in \mathbb{N},\right. \\
\left(T_{c}(t)(x)=T_{c}(t)(b)=\text { true }\right. \\
\text { and } \overline{T_{c}(t)(b)}=\text { true } \\
\left.\text { and } T_{c}(t)(y)=\text { true }\right) \text { or } \\
\left(T_{c}(t)(x)=T_{c}(t)(b)=\text { true }\right. \\
\text { and } \overline{T_{c}(t)(b)}=\text { false } \\
\left.\text { and } T_{c}(t)(y)=\text { false }\right) \text { or } \\
\left(T_{c}(t)(x)=T_{c}(t)(y)=\text { false }\right) \text { or } \\
\left.\left(T_{c}(t)(b)=T_{c}(t)(y)=\text { false }\right)\right\}\end{array}$ \\
\hline$y:=x$ default $z$ & $\begin{array}{c}\left\{T_{c} \in \mathcal{T} c_{\{x, y, z\}} \mid \forall t \in \mathbb{N}\right. \\
\left(T_{c}(t)(x)=T_{c}(t)(y)=\text { true }\right) \text { or } \\
\left(T_{c}(t)(x)=\text { false and }\right. \\
\left.\left.T_{c}(t)(z)=T_{c}(t)(y)\right)\right\}\end{array}$ \\
\hline$P_{1} \mid P_{2}$ & $\begin{array}{c}\left\{T_{c} \in \mathcal{T} c_{X_{1} \cup X_{2}} \mid\right. \\
\left.X_{1} \cdot T_{c} \in\left[\left[P_{1}\right]\right]_{c} \text { and } X_{2} \cdot T_{c} \in\left[\left[P_{2}\right]\right]_{c}\right\} \\
\text { where }\left[\left[P_{1}\right]\right]_{c} \subseteq \mathcal{T} c_{X_{1}},\left[\left[P_{2}\right]\right]_{c} \subseteq \mathcal{T} c_{X_{2}}\end{array}$ \\
\hline
\end{tabular}

Table 2 Clock Semantics of the Basic Processes

instant at which $x$ is present, $b$ is present and $b$ holds the value true, then $y$ cannot be set before $x$. We can use a Boolean condition $\hat{x} \wedge \hat{b} \wedge \bar{b}$ to encode the fact that $x$ is present, $b$ is present and $b$ holds the value true, where $\hat{x}, \hat{b}, \bar{b}$ are Boolean variables. Thus, the value of $y$ depends on the current value of $x$ whenever the condition $\hat{x} \wedge \hat{b} \wedge \bar{b}$ is satisfied.

In presenting the construction of a SDG below, first, we show that a usual Data Dependence Graph (DDG) is not sufficient to represent the dependences in a polychronous program. Then clock constraints are represented as first-order logic formulas as in Section 3.

\subsection{Data Dependence Graphs}

As in [4], a DDG is a directed graph which contains nodes that represent locations of definitions and uses of variables in basic blocks, and edges that represent data dependences between nodes. Considering the pseudo-code of a program called Sum, Figure 2 partially shows its DDG (the figure shows only the data dependences that are related to variable i). Data dependence edges are depicted by dotted lines which are added to the Control Flow Graph (CFG), and labelled by the name of the variable that creates the dependence. Node numbers in the CFG correspond to statement numbers in the program (we treat each statement as a basic block). Each node that represents a transfer of control (e.g., node 4) has 
two edges with labels $T$ (true) and $F$ (false), all others are unlabeled.

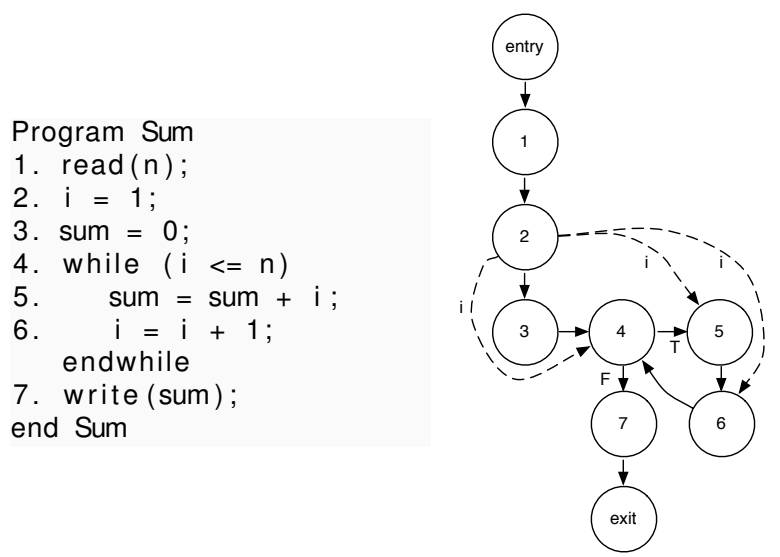

Fig. 2 CFG for Sum, with data dependence edges for i (dotted lines)

\subsection{Signal Program as Synchronous Dependence Graph}

Such data dependence graphs would not really represent the data dependences of a Signal program. Indeed, the dependences between signals in the program are not static. Since the presence of signals may vary along time (which is expressed by their clock), dependences also vary. To deal with that, the dependences are conditioned, and the conditions are represented by the clocks at which the dependences are effective.

To illustrate the definition of SDGs, we consider a process which involves the basic process corresponding to the deterministic merge operator:

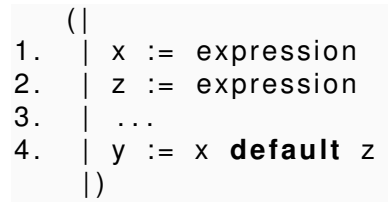

Here, the numbers are added only for documenting, and the statement number 3 denotes a segment of program. The statements 1,2 and 4 represent the expressions defining the signal $x, z$ and $y$, respectively. Roughly speaking, the signal $x$ is defined at statement 1 and is fetched at statement 4 . Considering the basic process $y:=x$ default $z$ (and the clock constraints between signals), the "valid" states are: $x$ is present and $y$ is present; or $x$ is absent, $z$ is present, and $y$ is present; or $x, y$ and $z$ are absent. They can be represented by $\hat{y} \Leftrightarrow(\hat{x} \vee \hat{z})$ in our Boolean abstraction. According to the valid states of the signals, the different data dependences between signals in the basic process $y:=x$ default $z$ are depicted in Figure 3, left, where the labels represent the conditions at which the dependences are effective. For instance, when $\hat{x}=$ true, $y$ is defined by $x$; otherwise it is defined by $z$ when $\hat{x}=$ false and $\hat{z}=$ true. We can see that the graph in this figure has the following property: an edge cannot exist if one of its extremity nodes is not present (or the corresponding signal holds no value). In our example, this property can be translated in the Boolean abstraction of clock semantics as: $\hat{x} \Rightarrow \hat{y} \wedge \hat{x}$ and $\neg \hat{x} \wedge \hat{z} \Rightarrow \hat{y} \wedge \hat{z}$.

A SDG for a given program is a labelled directed graph in which each node is a signal or clock variable and each edge represents the dependence between nodes. Each edge is labelled by a first-order logic formula over Boolean variables which represents the clock at which the dependence between the extremity nodes is effective. Formally, a SDG is defined as follows:

Definition 5. (SDG) A SDG associated with a process $P$ is a tuple $G=\left\langle N, E, I, O, C, m_{N}, m_{E}\right\rangle$ where:

- $N$ is a finite set of nodes, each of which represents the equation defining a signal or a clock;

- $E \subseteq N \times N$ is the set of dependences between nodes;

- $I \subseteq N$ is the set of input nodes;

- $O \subseteq N$ is the set of output nodes;

- $C$ is the set of Boolean formulas over a set of clocks in the Boolean abstraction;

- $m_{N}: N \longrightarrow C$ is a mapping labelling each node with a clock; it defines the existence condition of a node;

- $m_{E}: E \longrightarrow C$ is a mapping labeling each edge with a clock constraint; it defines the existence condition of an edge.

In contrast with DDG, the clock labelling in SDG provides a dynamic dependence feature. This clock labelling imposes two properties which are implicit for a SDG:

- An edge exists if its two extremity nodes exist. This property can be translated in our Boolean abstraction as:

$$
\forall(x, y) \in E, m_{E}(x, y) \Leftarrow\left(m_{N}(x) \wedge m_{N}(y)\right)
$$

- A cycle of dependences stands for a deadlock. It can be expressed as:

$$
\begin{aligned}
& \text { A SDG } \mathrm{G} \text { is deadlock-free iff } \\
& \forall x_{1}, \ldots, x_{n}, x_{1} \in G, \\
& m_{E}\left(x_{1}, x_{2}\right) \wedge m_{E}\left(x_{2}, x_{3}\right) \wedge \ldots \wedge m_{E}\left(x_{n}, x_{1}\right) \text { is false }
\end{aligned}
$$

We denote the fact that there exists a dependence between two nodes (signals or clocks) $x$ and $y$ at a clock constraint $m_{E}(x, y)=\hat{c}$ by $x \stackrel{\hat{c}}{\rightarrow} y$. A dependence path from $x$ to $y$ is any 


\begin{tabular}{|c|c|}
\hline Process $P$ & Clock Semantics $[[P]]_{c}$ \\
\hline$y:=f\left(x_{1}, \ldots, x_{n}\right)$ & $\begin{array}{c}\left\{T_{c} \in \mathcal{T} c_{\left\{y, x_{1}, \ldots, x_{n}\right\}} \mid \forall t \in \mathbb{N},\right. \\
\left(\forall i, T_{c}(t)\left(x_{i}\right)=T_{c}(t)(y)\right)=\text { false or } \\
\left(\forall i, T_{c}(t)\left(x_{i}\right)=T_{c}(t)(y)=\text { true and }\right. \\
\left.\left.\overline{T_{c}(t)(y)}=f\left(\overline{T_{c}(t)\left(x_{1}\right)}, \ldots, \overline{T_{c}(t)\left(x_{n}\right)}\right)\right)\right\}\end{array}$ \\
\hline$y:=x \$ 1$ init $a$ & $\begin{array}{c}\left\{T_{c} \in \mathcal{T} c_{\{x, y\}} \mid \forall t \in \mathbb{N},\right. \\
\left(T_{c}(t)(x)=T_{c}(t)(y)\right) \text { or } \\
\left(T_{c}(t)(x)=T_{c}(t)(y)=\text { true and }\right. \\
\overline{T_{c}\left(t_{0}\right)(y)}=a \text { and } \\
\left.\forall t \geq t_{0}, \overline{T_{c}(t)(y)}=\overline{T_{c}\left(t_{-}\right)(x)}\right) \\
\text { with } t_{0}=\inf \left\{t^{\prime} \mid T_{c}\left(t^{\prime}\right)(x)=\text { true }\right\} \\
\left.t_{-}=\sup \left\{t^{\prime} \mid t^{\prime}<t \wedge T_{c}\left(t^{\prime}\right)(x)=\text { true }\right\}\right\}\end{array}$ \\
\hline$y:=x$ when $b$ & $\begin{array}{c}\left\{T_{c} \in \mathcal{T} c_{\{x, y, b\}} \mid \forall t \in \mathbb{N}\right. \\
\left(T_{c}(t)(x)=T_{c}(t)(b)=\text { true }\right. \\
\text { and } \overline{T_{c}(t)(b)}=\text { true } \\
\text { and } T_{c}(t)(y)=\text { true } \text { and } \\
\left.\overline{T_{c}(t)(y)}=\overline{T_{c}(t)(x)}\right) \text { or } \\
\left(T_{c}(t)(x)=T_{c}(t)(b)=\text { true }\right. \\
\text { and } \overline{T_{c}(t)(b)}=\text { false } \\
\text { and } T_{c}(t)(y)=\text { false } \text { or } \\
\left(T_{c}(t)(x)=T_{c}(t)(y)=\text { false }\right) \text { or } \\
\left.\left(T_{c}(t)(b)=T_{c}(t)(y)=\text { false }\right)\right\}\end{array}$ \\
\hline$y:=x$ default $z$ & $\begin{array}{c}\left\{T_{c} \in \mathcal{T} c_{\{x, y, z\}} \mid \forall t \in \mathbb{N},\right. \\
\left(T_{c}(t)(x)=T_{c}(t)(y)=\text { true and }\right. \\
\left.\overline{T_{c}(t)(y)}=\overline{T_{c}(t)(x)}\right) \text { or } \\
\left(T_{c}(t)(x)=\text { false and } T_{c}(t)(y)=T_{c}(t)(z)\right. \\
\left.\left.\text { and } \overline{T_{c}(t)(y)}=\overline{T_{c}(t)(z)}\right)\right\}\end{array}$ \\
\hline$P_{1} \mid P_{2}$ & $\begin{array}{c}\left\{T_{c} \in \mathcal{T} c_{X_{1} \cup X_{2}} \mid\right. \\
\left.X_{1} . T_{c} \in\left[\left[P_{1}\right]\right]_{c} \text { and } X_{2} . T_{c} \in\left[\left[P_{2}\right]\right]_{c}\right\} \\
\text { where }\left[\left[P_{1}\right]\right]_{c} \subseteq \mathcal{T} c_{X_{1}},\left[\left[P_{2}\right]\right]_{c} \subseteq \mathcal{T} c_{X_{2}}\end{array}$ \\
\hline
\end{tabular}

Table 3 Clock Semantics of the Basic Processes with Boolean Signals

set of nodes $s=\left\{x_{0}, x_{1}, \ldots, x_{k}\right\}$ such that (an edge is a special case when $k=1$ ):

$$
x=x_{0} \stackrel{\widehat{c_{0}}}{\longrightarrow} x_{1} \stackrel{\widehat{c_{1}}}{\longrightarrow} \ldots \stackrel{\widehat{c_{k-1}}}{\longrightarrow} x_{k}=y
$$

In Table 4, we construct the dependences between signals for the core language, where the subclocks $[c]$ and $[\neg c]$ are encoded as $\hat{c} \wedge \bar{c}$ and $\hat{c} \wedge \neg \bar{c}$, respectively, in our abstraction. The edges are labelled by clocks which are represented by a Boolean formula in our abstraction. All the dependences in this table impose the implicit properties for a SDG, for instance, the basic process of the primitive operator Boolean sampling satisfies that $\hat{y} \Rightarrow \hat{x} \wedge \hat{y}$ and $\hat{y} \Rightarrow \hat{b} \wedge \hat{y}$. We also assume that all considered programs are written with the primitive operators, meaning that derived operators are replaced by their definition with primitive ones, and there are no nested operators (these nested operators can be broken by using fresh signals). Following the above construction rules, we can obtain the SDG in Figure 3, right, for the simple pro-

\begin{tabular}{|c|c|}
\hline$x$ & $\begin{array}{l}C_{x} \stackrel{\hat{x}}{\rightarrow} x \\
m_{N}\left(C_{x}\right)=\hat{x}, m_{N}(x)=\hat{x}\end{array}$ \\
\hline$c$ (Boolean signal) & $\begin{array}{l}c \stackrel{\hat{c}}{\rightarrow}[c] \\
m_{N}(c)=\hat{c}, m_{N}([c])=\hat{c} \\
c \stackrel{\hat{c}}{\rightarrow}[\neg c] \\
m_{N}(c)=\hat{c}, m_{N}([\neg c])=\hat{c}\end{array}$ \\
\hline$x \stackrel{c}{\rightarrow} y$ & $\begin{array}{l}{[c] \stackrel{\hat{c}}{\rightarrow} y} \\
m_{N}([c])=\hat{c}, m_{N}(y)=\hat{y}\end{array}$ \\
\hline$y:=f\left(x_{1}, \ldots, x_{n}\right)$ & $\begin{array}{l}x_{1} \stackrel{\hat{y}}{\rightarrow} y \\
\ldots \\
x_{n} \stackrel{\hat{y}}{\rightarrow} y \\
m_{N}\left(x_{i}\right)=\widehat{x}_{i}, m_{N}(y)=\hat{y}, i=1, \ldots, n\end{array}$ \\
\hline$y:=x \$ 1$ init $a$ & $\begin{array}{l}y \stackrel{\hat{y}}{\rightarrow} x \\
m_{N}(y)=\hat{y}, m_{N}(x)=\hat{x}\end{array}$ \\
\hline$y:=x$ when $b$ & $\begin{array}{l}x \stackrel{\hat{y}}{\rightarrow} y \\
m_{N}(x)=\hat{x}, m_{N}(y)=\hat{y} \\
b \stackrel{\hat{y}}{\rightarrow} C_{y} \\
m_{N}(b)=\hat{b}, m_{N}\left(C_{y}\right)=\hat{y}\end{array}$ \\
\hline$y:=x$ default $z$ & $\begin{array}{l}x \stackrel{\hat{x}}{\rightarrow} y \\
m_{N}(x)=\hat{x}, m_{N}(y)=\hat{y} \\
z \stackrel{\hat{z} \wedge \neg \hat{x}}{\longrightarrow} y \\
m_{N}(z)=\hat{z}, m_{N}(y)=\hat{y}\end{array}$ \\
\hline
\end{tabular}

Table 4 The Dependences of the Core Language

gram DEC (we omit the part of graph that represents the dependences between $Z N_{2}, Z N_{1}$ and $Z N$ ).

\section{Translation Validation for Synchronous Pro- gram Transformations}

We adopt the translation validation approach $[33,34]$ to formally verify that the clock semantic and the dependence between variables in the program are preserved for every transformation of the compiler. To do that our verification framework uses clock models to represent the clock semantic of original program and its transformed counterpart. Then we introduce a refinement relation which expresses the preservations of clock semantic, as relation on clock models. Thus, if $\Phi\left(P_{1}\right)$ and $\Phi\left(P_{2}\right)$ are clock models, $\Phi\left(P_{2}\right) \sqsubseteq_{c l k} \Phi\left(P_{1}\right)$ means that $\Phi\left(P_{2}\right)$ is a refinement of $\Phi\left(P_{1}\right)$. This relation could be interpreted to mean that if a clock trace is in the set of clock traces of $\Phi\left(P_{2}\right)$, then it belongs to the set of clock traces of $\Phi\left(P_{1}\right)$ as well. For the preservation of dependence, we use SDGs to represent the dependence in original program and its transformed counterpart. A refinement relation which expresses the preservation of dependences between signals 


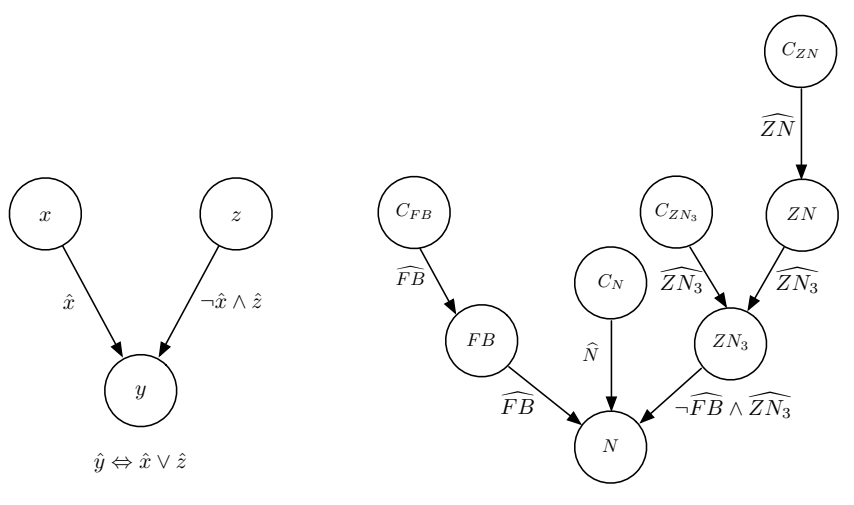

Fig. 3 The SDG Example and SDG of DEC

is defined as a relation on synchronous dependence graphs. Given $S D G\left(P_{1}\right)$ and $S D G\left(P_{2}\right), S D G\left(P_{2}\right) \sqsubseteq_{\text {dep }} S D G\left(P_{1}\right)$ means that $S D G\left(P_{2}\right)$ is a refinement of $S D G\left(P_{1}\right)$. This relation could be interpreted to mean that if there exists a dependence path from signal $x$ to signal $y$ in $S D G\left(P_{1}\right)$ under the clock constraint $\widehat{c_{1}}$, then there is a dependence path from $x$ to $y$ in $S D G\left(P_{2}\right)$ under the clock constraint $\widehat{c_{2}}$ such that whenever the dependence path in $S D G\left(P_{1}\right)$ is effective, the dependence path in $S D G\left(P_{2}\right)$ is effective too, or $\widehat{c_{1}} \Rightarrow \widehat{c_{2}}$. And $S D G\left(P_{2}\right)$ introduces no deadlocks.

\subsection{Translation Validation for Clock Transformations}

\subsubsection{Definition of Correct Transformation: Clock Refine- ment}

Let $\Phi\left(P_{1}\right)$ and $\Phi\left(P_{2}\right)$ be two clock models, to which we refer respectively as a source program and its transformed counterpart produced by the compiler. We assume that they have the same set of input and output variables. We will discuss in detail in the next section in case the compiler renames some local variables. We say that $P_{1}$ and $P_{2}$ have the same clock semantics if $\Phi\left(P_{1}\right)$ and $\Phi\left(P_{2}\right)$ have the same set of clock traces:

$$
\forall T_{c} .\left(T_{c} \in \Gamma\left(\Phi\left(P_{1}\right)\right) \Leftrightarrow T_{c} \in \Gamma\left(\Phi\left(P_{2}\right)\right)\right)
$$

In general, the compilation makes the transformed program more concrete. For instance, when the Signal compiler do the Boolean abstraction which is used to generate the sequential executable code, the signal with the fastest clock is always present in the generated code. Additionally, compilers do transformations, optimizations for removing or eliminating some redundant behaviors of the source program (e.g. eliminating subexpressions, trivial clock relations). Therefore, Requirement (6) is too strong to be practical. To address this issue, we relax the requirement as follows:

$$
\forall T_{c} .\left(T_{c} \in \Gamma\left(\Phi\left(P_{2}\right)\right) \Rightarrow T_{c} \in \Gamma\left(\Phi\left(P_{1}\right)\right)\right)
$$

Requirement (7) expresses that if every clock trace of $\Phi\left(P_{2}\right)$ is also a clock trace of $\Phi\left(P_{1}\right)$, or $\Gamma\left(\Phi\left(P_{2}\right)\right) \subseteq \Gamma\left(\Phi\left(P_{1}\right)\right)$. We say that $\Phi\left(P_{2}\right)$ is a correct clock transformation of $\Phi\left(P_{1}\right)$ or $\Phi\left(P_{2}\right)$ is a clock refinement of $\Phi\left(P_{1}\right)$.

Proposition 2. The clock refinement is reflexive and transitive, or:

- $\forall \Phi(P), \Phi(P) \sqsubseteq_{c l k} \Phi(P)$.

- If $\Phi\left(P_{1}\right) \sqsubseteq_{c l k} \Phi\left(P_{2}\right)$ and $\Phi\left(P_{2}\right) \sqsubseteq_{c l k} \Phi\left(P_{3}\right)$, then $\Phi\left(P_{1}\right) \sqsubseteq_{c l k} \Phi\left(P_{3}\right)$.

Proof. The reflexivity is obvious based on the clock refinement definition. For every clock trace $T_{c} \in \Gamma\left(\Phi\left(P_{1}\right)\right)$, then $T_{c} \in \Gamma\left(\Phi\left(P_{2}\right)\right)$. Since $\Phi\left(P_{2}\right) \sqsubseteq_{c l k} \Phi\left(P_{3}\right)$, we have $T_{c} \in$ $\Gamma\left(\Phi\left(P_{3}\right)\right)$, or $\Phi\left(P_{1}\right) \sqsubseteq_{c l k} \Phi\left(P_{3}\right)$.

\subsubsection{Proving Clock Refinement by SMT solver}

We now discuss an approach to check the existence of refinement between two clock models (Requirement (7)) which is based on the following theorem.

Theorem 1. Given a source program $P_{1}$ and its transformed program $P_{2}, P_{2}$ is a correct clock transformation of $P_{1}$ if it satisfies that for every interpretation $\hat{I}$, if $\hat{I}$ is a clock configuration of $\Phi\left(P_{2}\right)$ then it is a clock configuration of $\Phi\left(P_{1}\right)$, or:

$$
\left(\vDash \Phi\left(P_{2}\right) \Rightarrow \Phi\left(P_{1}\right)\right) \Rightarrow \Phi\left(P_{2}\right) \sqsubseteq_{c l k} \Phi\left(P_{1}\right)
$$

Proof. To prove Theorem 1, we show that if $\forall \hat{I} .(\hat{I} \vDash$ $\left.\left.\Phi\left(P_{2}\right) \Rightarrow \hat{I} \models \Phi_{(} P_{1}\right)\right)$ then $\Gamma\left(\Phi\left(P_{2}\right)\right) \subseteq \Gamma\left(\Phi\left(P_{1}\right)\right)$. Given $T_{c} \in$ $\Gamma\left(\Phi\left(P_{2}\right)\right)$, it means that $\forall t, T_{c}(t) \in S_{\text {sat }}\left(\Phi\left(P_{2}\right)\right)$. Since $\forall \hat{I} .(\hat{I} \models$ $\left.\Phi\left(P_{2}\right) \Rightarrow \hat{I} \vDash \Phi\left(P_{1}\right)\right)$, thus $S_{\text {sat }}\left(\Phi\left(P_{2}\right)\right) \subseteq S_{\text {sat }}\left(\Phi\left(P_{1}\right)\right)$, meaning that $T_{c}(t) \in S_{s a t}\left(\Phi\left(P_{1}\right)\right)$ for every $t$. Therefore, we have $T_{c} \in \Gamma\left(\Phi\left(P_{1}\right)\right)$.

To solve the validity of the formula $\left(\Phi\left(P_{2}\right) \Rightarrow \Phi\left(P_{1}\right)\right)$ in (8), a SMT solver is needed since this formula involves nonBoolean variables and uninterpreted functions. A SMT solver decides the satisfiability of arbitrary logic formulas of linear real and integer arithmetic, scalar types, other user-defined data structures, and uninterpreted functions. If the formula belongs to the decidable theory, the solver gives two types of answers: sat when the formula has a model (there exists an interpretation that satisfies it); or unsat otherwise. In 
our case, we will ask the solver to check whether the formula $\neg\left(\Phi\left(P_{2}\right) \Rightarrow \Phi\left(P_{1}\right)\right)$ is unsatisfiable. Since $\neg\left(\Phi\left(P_{2}\right) \Rightarrow\right.$ $\left.\Phi\left(P_{1}\right)\right)$ is unsatisfiable iff $\left.\models \Phi\left(P_{2}\right) \Rightarrow \Phi\left(P_{1}\right)\right)$.

In our translation validation, the clock models which are constructed from Boolean, numerical variables and uninterpreted functions belong to a part of first-order logic which have a small model property according to [?]. The numerical variables are involved only in some implication with uninterpreted functions such as $\left(\bar{x}=\overline{x^{\prime}} \wedge \bar{y}=\overline{y^{\prime}}\right) \Rightarrow \overline{v_{\square}^{i}}=\overline{v_{\square}^{j}}$. In addition, the formula is quantifier-free. This means the check of satisfiability can be established by examining a certain finite cardinality of models, and it can be solved efficiently and significantly improves the scalability of the solver.

\subsubsection{Illustrate on Example}

Consider the program $\mathrm{DEC}$ and its transformed program of the clock calculation phase of the Signal compiler, DEC_BASIC_TRA. For the validation process, the clock semantics of the transformed program is also represented as the clock model, $\Phi($ DEC_BASIC_TRA) as follows:

$$
\begin{aligned}
& \left(\widehat{C L K} \Leftrightarrow C \widehat{L K \_} \wedge \neg C \widehat{C L K \_} \widehat{F B}\right) \wedge(\widehat{C L K} \Rightarrow \overline{C L K}) \\
& \wedge\left(C \widehat{L K \_} N \Leftrightarrow C \widehat{L K_{-}} N \vee C \widehat{L K \_F B}\right) \\
& \wedge\left(C \widehat{L K_{-}} N \Rightarrow \overline{C L K_{-} N}\right) \\
& \wedge C \widehat{L K \_} \Leftrightarrow \widehat{N} \Leftrightarrow \widehat{Z N} \\
& \wedge\left(\widehat{N} \Leftrightarrow \widehat{F B} \wedge C \widehat{L K_{-} F B} \vee \widehat{v_{-}^{1}} \wedge \widehat{C L K}\right) \\
& \wedge\left(\widehat{v_{-}^{1}} \Leftrightarrow \widehat{Z N}\right) \\
& \wedge(\widehat{Z N} \Leftrightarrow \widehat{N})
\end{aligned}
$$

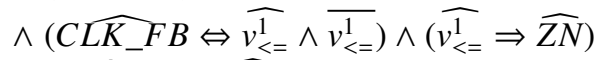

$$
\begin{aligned}
& \wedge C \widehat{L K \_F B} \Leftrightarrow \widehat{F B} \\
& \wedge C \widehat{L K_{-}} 12 \Leftrightarrow \widehat{v_{<=}^{1}} \wedge \neg \overline{v_{<=}^{1}}
\end{aligned}
$$

Then to check the transformation from $\mathrm{DEC}$ to DEC_BASIC_TRA is correct w.r.t the clock semantics, the validator will solve the validity of the formula $\Phi($ DEC_BASIC_TRA $) \Rightarrow \Phi($ DEC $)$.

\subsection{Translation Validation for SDGs}

\subsubsection{Definition of Correct Transformation: Dependence Refinement}

Considering two SDGs $S D G\left(P_{1}\right)$ and $S D G\left(P_{2}\right)$, to which we refer respectively as a source program and its transformed counterpart produced by a compiler. A dependence path from $x$ to $y$ in $S D G\left(P_{2}\right)$ is reinforcement of the dependence path from $x$ to $y$ in $S D G\left(P_{1}\right)$ if at any instant the dependence path in $S D G\left(P_{1}\right)$ is effective implying that the dependence path in $S D G\left(P_{2}\right)$ is effective.
Definition 6. (Reinforcement) Let $d p_{1}=x \stackrel{\widehat{c_{0}}}{\longrightarrow} x_{1} \stackrel{\widehat{c_{1}}}{\longrightarrow} \ldots \stackrel{\widehat{c_{n-1}}}{\longrightarrow}$ $y$ and $d p_{2}=x \stackrel{\widehat{c_{0}^{\prime}}}{\longrightarrow} x_{1}^{\prime} \stackrel{\widehat{c_{1}^{\prime}}}{\longrightarrow} \ldots \stackrel{\widehat{c_{m-1}^{\prime}}}{\longrightarrow} y$ be two dependence paths in $S D G\left(P_{1}\right)$ and $S D G\left(P_{2}\right)$, respectively. It is said that $d p_{2}$ is a reinforcement of $d p_{1}$ iff $\left(\bigwedge_{i=0}^{n-1} \widehat{c_{i}} \Rightarrow \bigwedge_{j=0}^{m-1} \widehat{c_{j}^{\prime}}\right)$.

We write $d p_{2} \leq_{d e p} d p_{1}$ to denote the fact that $d p_{2}$ is a reinforcement of $d p_{1}$. The condition $\left(\bigwedge_{i=0}^{n-1} \widehat{c_{i}} \Rightarrow \bigwedge_{j=0}^{m-1} \widehat{c_{j}^{\prime}}\right)$ is used to indicate that if the dependence path in $S D G\left(P_{1}\right)$ is effective then the dependence path in $S D G\left(P_{2}\right)$ is effective. In the special case when $m=n=1, x \stackrel{\widehat{c_{0}^{\prime}}}{\rightarrow} y$ is a reinforcement of $x \stackrel{\widehat{c_{0}}}{\longrightarrow} y$ iff $\left(c_{0} \Rightarrow c_{0}^{\prime}\right)$.

Definition 7. (Deadlock consistency) A dependence path $d p_{2}=x \stackrel{\widehat{c_{0}^{\prime}}}{\rightarrow} x_{1}^{\prime} \stackrel{\widehat{c_{1}^{\prime}}}{\rightarrow} \ldots \stackrel{\widehat{c_{m-1}^{\prime}}}{\longrightarrow} y$ in $S D G\left(P_{2}\right)$ is a deadlockconsistent for $d p_{1}=x \stackrel{\widehat{c_{0}}}{\rightarrow} x_{1} \stackrel{\widehat{c_{1}}}{\rightarrow} \ldots \stackrel{\widehat{c_{n-1}}}{\longrightarrow} y$ in $S D G\left(P_{1}\right)$ iff for every dependence path $y \stackrel{\widehat{l_{0}}}{\rightarrow} z_{1} \stackrel{\widehat{l_{1}}}{\rightarrow} \ldots \stackrel{\widehat{l_{p-1}}}{\rightarrow} x$ in $S D G\left(P_{1}\right)$ such that $\left(\bigwedge_{i=0}^{n-1} \widehat{c_{i}} \wedge \bigwedge_{j=0}^{p-1} \widehat{l_{j}}\right) \Leftrightarrow$ false, then for every dependence path $y \stackrel{\widehat{l_{0}^{\prime}}}{\rightarrow} z_{1}^{\prime} \stackrel{\widehat{l_{1}^{\prime}}}{\rightarrow} \ldots \stackrel{\widehat{l_{q-1}^{\prime}}}{\rightarrow} x$ in $S D G\left(P_{2}\right)$, it satisfies $\left(\bigwedge_{u=0}^{m-1} \widehat{c_{u}^{\prime}} \wedge \bigwedge_{v=0}^{q-1} \widehat{l_{u}^{\prime}}\right) \Leftrightarrow$ false, denoted by $d p_{2} \asymp_{\text {dep }} d p_{1}$.

When $m=n=p=q=1, x \stackrel{\widehat{c_{0}^{\prime}}}{\rightarrow} y$ is deadlock-consistent for $x \stackrel{\widehat{c_{0}}}{\rightarrow} y$ iff $\left(\left(c_{0} \wedge l_{0}\right) \Leftrightarrow\right.$ false $) \Rightarrow\left(\left(c_{0}^{\prime} \wedge l_{0}^{\prime}\right) \Leftrightarrow\right.$ false $)$. Deadlock consistency expresses the fact that if there are dependence paths from a signal $x$ to a signal $y$ and vise-versa such that there is no cyclic dependence between $x$ and $y$ in the source program, then the transformed program cannot introduce any cyclic dependence between $x$ and $y$.

Recall that $S D G\left(P_{1}\right)$ and $S D G\left(P_{2}\right)$ are two SDGs, we assume that they have the same set of nodes. We say that the transformed counterpart $P_{2}$ of the source program $P_{1}$ preserves the dependences between signals if the following conditions are satisfied:

1. For any dependence path between signals from signal $x$ to signal $y$ in $S D G\left(P_{1}\right)$ at a clock constraint $\widehat{c_{1}}$, then there exists a dependence path from $x$ to $y$ at a clock constraint $\widehat{c_{2}}$ in $S D G\left(P_{2}\right)$ such that whenever the dependence in $S D G\left(P_{1}\right)$ is effective, then the dependence in $S D G\left(P_{2}\right)$ is also effective.

2. If there is no deadlocks in $S D G\left(P_{1}\right)$, then $S D G\left(P_{2}\right)$ introduces no deadlocks

We say that $S D G\left(P_{2}\right)$ is a correct transformation of $S D G\left(P_{1}\right)$ or $S D G\left(P_{2}\right)$ is a dependence refinement of $S D G\left(P_{1}\right)$. We write $S D G\left(P_{2}\right) \sqsubseteq_{d e p} S D G\left(P_{1}\right)$ to denote the fact that there exists a dependence refinement relation between $S D G\left(P_{2}\right)$ and $S D G\left(P_{1}\right)$. The formal definition of dependence refinement is: 
Definition 8. (Dependence refinement) Let $S D G\left(P_{1}\right)$ and $S D G\left(P_{2}\right)$ be two synchronous dependence graphs, $S D G\left(P_{2}\right)$ is a dependence refinement of $S D G\left(P_{1}\right)$ if:

$$
\begin{aligned}
& \text { - } \forall d p_{1}=x \stackrel{\widehat{c_{0}}}{\longrightarrow} x_{1} \underset{c_{1}}{\longrightarrow} \ldots \stackrel{\widehat{c_{n-1}}}{\longrightarrow} y \text { in } S D G\left(P_{1}\right) \text {, } \\
& \exists d p_{2}=x \stackrel{\widehat{c_{0}^{\prime}}}{\longrightarrow} x_{1}^{\prime} \stackrel{\widehat{c_{1}^{\prime}}}{\longrightarrow} \ldots \stackrel{\widehat{c_{m-1}^{\prime}}}{\longrightarrow} y \text { in } S D G\left(P_{2}\right) \\
& \text { s.t } d p_{2} \preceq_{d e p} d p_{1} \\
& \text { - } \forall d p_{1}=x \stackrel{\widehat{c_{0}}}{\longrightarrow} x_{1} \stackrel{\widehat{c_{1}}}{\longrightarrow} \ldots \stackrel{\widehat{c_{n-1}}}{\longrightarrow} y \text { in } S D G\left(P_{1}\right) \text { and } \\
& \forall d p_{2}=x \stackrel{\widehat{c_{0}^{\prime}}}{\rightarrow} x_{1}^{\prime} \stackrel{\widehat{c_{1}^{\prime}}}{\rightarrow} \ldots \stackrel{\widehat{c_{m-1}^{\prime}}}{\longrightarrow} y \text { in } \operatorname{SDG}\left(P_{2}\right) \text {, } \\
& d p_{2} \asymp_{\text {dep }} d p_{1}
\end{aligned}
$$

Proposition 3. The reinforcement, deadlock consistency, and dependence refinement are reflexive and transitive.

\section{Proof. Reinforcement}

- Reflexivity: For any dependence path $d p$, based on the definition, we always have $d p \preceq_{d e p} d p$.

- Transitivity: Assume that $d p_{1} \unlhd_{d e p} d p_{2}$ and $d p_{2} \unlhd_{d e p}$ $d p_{3}$, we have $\left(\bigwedge_{i=0}^{n-1} \widehat{c_{i}} \Rightarrow \bigwedge_{j=0}^{m-1} \widehat{c_{j}^{\prime}}\right) \wedge\left(\bigwedge_{j=0}^{m-1} \widehat{c_{j}^{\prime}} \Rightarrow\right.$ $\left.\bigwedge_{k=0}^{p-1} \widehat{c^{\prime \prime}}{ }_{k}\right)$, thus $\left(\bigwedge_{i=0}^{n-1} \widehat{c_{i}} \Rightarrow \bigwedge_{k=0}^{p-1} \widehat{c^{\prime \prime}{ }_{k}}\right)$, or $d p_{1} \unlhd_{d e p} d p_{3}$.

\section{Deadlock consistency}

- Reflexivity: Based on the definition, we always have $d p \asymp_{d e p} d p$.

- Transitivity: Assume that $d p_{1} \asymp_{d e p} d p_{2}$ and $d p_{2} \asymp_{d e p}$ $d p_{3}$, we have $\left(\left(\left(\bigwedge_{i=0}^{n-1} \widehat{c_{i}} \wedge \wedge_{j=0}^{p-1} \widehat{l_{j}}\right) \Leftrightarrow\right.\right.$ false $) \Rightarrow$ $\left(\left(\bigwedge_{u=0}^{m-1} \widehat{c_{u}^{\prime}} \wedge \bigwedge_{v=0}^{q-1} \widehat{l_{u}^{\prime}}\right) \Leftrightarrow\right.$ false $) \wedge\left(\left(\bigwedge_{u=0}^{m-1} \widehat{c_{u}^{\prime}} \wedge \bigwedge_{v=0}^{q-1} \widehat{l_{u}^{\prime}}\right) \Leftrightarrow\right.$ false $) \Rightarrow\left(\left(\bigwedge_{t=0}^{r-1} \widehat{c}_{t} \wedge \bigwedge_{z=0}^{s-1} \widehat{l}_{z}\right) \Leftrightarrow\right.$ false $\left.)\right)$, thus $\left(\left(\bigwedge_{i=0}^{n-1} \widehat{c_{i}} \wedge \wedge_{j=0}^{p-1} \widehat{l_{j}}\right) \Leftrightarrow\right.$ false $) \Rightarrow\left(\left(\bigwedge_{t=0}^{r-1} \widehat{c^{\prime \prime}}{ }_{t} \wedge\right.\right.$ $\left.\bigwedge_{z=0}^{s-1} \widehat{l}_{z}\right) \Leftrightarrow$ false)), or $d p_{1} \asymp_{d e p} d p_{3}$.

\section{Dependence refinement}

- Reflexivity: For every dependence path $d p$ in $S D G(P)$, we have $d p \varsigma_{d e p} d p$ and $d p \asymp_{d e p} d p$, thus $S D G(P) \sqsubseteq_{d e p}$ $S D G(P)$.

- Transitivity: Assume that $S D G\left(P_{1}\right) \sqsubseteq_{d e p} S D G\left(P_{2}\right)$ and $S D G\left(P_{2}\right) \sqsubseteq_{d e p} S D G\left(P_{3}\right)$, we will show that $S D G\left(P_{1}\right)$ $\sqsubseteq_{\text {dep }} S D G\left(P_{3}\right)$.

i) For every dependence path $d p_{3}$ in $S D G\left(P_{3}\right)$, there exists a dependence path $d p_{2}$ in $S D G\left(P_{2}\right)$ such that $d p_{2} \preceq_{d e p} d p_{3}$. Since $S D G\left(P_{1}\right) \sqsubseteq_{d e p} S D G\left(P_{2}\right)$, there exists a dependence path $d p_{1}$ in $S D G\left(P_{1}\right)$ such that $d p_{1} \preceq_{d e p} d p_{2}$. Following the transitivity of the reinforcement, we have $d p_{1} \leq_{d e p} d p_{3}$.

ii) For every dependence path $d p_{1}$ and $d p_{2}$ from node $x$ to node $y$ in $S D G\left(P_{1}\right)$ and $S D G\left(P_{2}\right)$, respectively, it satisfies $d p_{1} \asymp_{d e p} d p_{2}$ since $S D G\left(P_{1}\right) \sqsubseteq_{d e p} S D G\left(P_{2}\right)$. Because $S D G\left(P_{2}\right) \sqsubseteq_{d e p} S D G\left(P_{3}\right)$, for every dependence path $d p_{3}$ from $x$ to $y$ in $S D G\left(P_{3}\right)$, we have $d p_{2} \asymp_{d e p} d p_{3}$. Apply the transitivity property of the deadlock consistency, we have $d p_{1} \asymp_{\text {dep }} d p_{3}$.

\subsubsection{Proving Dependence Refinement by SMT solver}

Given two SDGs, we introduce an approach to check the existence of dependence refinement between them that is implemented with a SMT-solver. A SMT-solver decides the satisfiability of arbitrary logic formulas of linear real and integer arithmetic, scalar types, other user-defined data structures, and uninterpreted functions. If the formula belongs to the decidable theory, the solver gives two types of answers: sat when the formula has a model (there exists an interpretation that satisfies it); or unsat otherwise. In our case, the formulas which label the edges of the graphs are over Boolean variables, thus the solving is decidable and very efficient [10].

Following Definition 8, we will traverse the entire graphs $S D G\left(P_{1}\right)$ and $S D G\left(P_{2}\right)$ to verify that:

- for every path in $S D G\left(P_{1}\right)$, there exists a reinforcement path in $S D G\left(P_{2}\right)$,

- and for any path from $x$ to $y$ in $S D G\left(P_{1}\right)$ and $S D G\left(P_{2}\right)$, they are deadlock-consistent.

It means that the basic element which is verified is that given two dependence paths, how we check the reinforcement and deadlock-consistent properties. Consider two dependence paths $d p_{1}=x \stackrel{\widehat{c_{0}}}{\longrightarrow} x_{1} \stackrel{\widehat{c_{1}}}{\longrightarrow} \ldots \stackrel{\widehat{c_{n-1}}}{\longrightarrow} y$ and $d p_{2}=$ $x \stackrel{\widehat{c_{0}^{\prime}}}{\rightarrow} x_{1}^{\prime} \stackrel{\widehat{c_{1}^{\prime}}}{\rightarrow} \ldots \stackrel{\widehat{c_{m-1}^{\prime}}}{\longrightarrow} y, d p_{2}$ is a reinforcement of $d p_{1}$ iff $\left(\bigwedge_{i=0}^{n-1} \widehat{c_{i}} \Rightarrow \bigwedge_{j=0}^{m-1} \widehat{c_{j}^{\prime}}\right)$. The checking of this condition can be implemented by asking a SMT-solver to check $\vDash$ $\left(\bigwedge_{i=0}^{n-1} \widehat{c_{i}} \Rightarrow \bigwedge_{j=0}^{m-1} \widehat{c_{j}^{\prime}}\right)$. In the same way, a SMT-solver can be used to check the deadlock consistency between two dependence paths, that means we will ask the SMT-solver to check the validity of the formula $\left(\left(\bigwedge_{i=0}^{n-1} \widehat{c_{i}} \wedge \bigwedge_{j=0}^{p-1} \widehat{l_{j}}\right) \Leftrightarrow\right.$ false $) \Rightarrow$ $\left(\left(\bigwedge_{u=0}^{m-1} \widehat{c_{u}^{\prime}} \wedge \bigwedge_{v=0}^{p-1} \widehat{l_{u}^{\prime}}\right) \Leftrightarrow\right.$ false $)$

We present here the concept of abstraction over SDGs which enable the checking process more efficient. According to the nature of SDGs, the abstraction is computed through the following rules of parallel and series [29] upon the input and output nodes:

$$
\begin{aligned}
& x \stackrel{\widehat{c_{0}}}{\longrightarrow} y \stackrel{\widehat{c_{1}}}{\longrightarrow} z \quad \Rightarrow x \stackrel{\widehat{c_{0}} \wedge \widehat{c_{1}}}{\longrightarrow} z \\
& x \stackrel{\widehat{c_{0}}}{\rightarrow} y \text { and } x \stackrel{\widehat{c_{1}}}{\longrightarrow} y \Rightarrow x \stackrel{\widehat{c_{0}} \vee \widehat{c_{1}}}{\longrightarrow} y
\end{aligned}
$$

Let $\overline{S D G\left(P_{1}\right)}$ and $\overline{S D G\left(P_{2}\right)}$ be graphs which are applied the rules of parallel and series, then $\overline{S D G\left(P_{2}\right)}$ is a dependence 
refinement of $\overline{S D G\left(P_{1}\right)}$ if the following conditions are satisfied:

$$
\begin{aligned}
& \text { - } \forall e_{1}=(x, y) \text { in } \overline{S D G\left(P_{1}\right)}, \exists e_{2}=(x, y) \text { in } \overline{S D G\left(P_{2}\right)} \\
& \quad \text { s.t } e_{2} \leftrightharpoons_{d e p} e_{1} \\
& \text { - } \forall e_{1}=(x, y) \text { in } \overline{S D G\left(P_{1}\right)}, e_{2}=(x, y) \text { in } \overline{S D G\left(P_{2}\right)} \\
& \quad e_{2} \asymp_{d e p} e_{1}
\end{aligned}
$$

\subsection{Toward Certified Compiler}

Given a program $P$, with an unverified compiler, we consider the following process:

1. The compiler takes program $P$ and transforms it.

2. If there is any error (i.e. syntax errors), it outputs an Error.

3. Otherwise, it outputs the intermediate representation $\operatorname{IR}(P)$ (i.e. the intermediate representation is written in the same language syntaxes as the source program $P$ ).

These steps can be represented in the following pseudo-code, where $C p(P)$ is the compilation step from the source program $P$ to either compiled code $I R(P)$ or compilation errors.

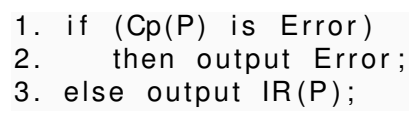

Now, it is followed by our refinement verification which checks that the transformed program $I R(P)$ refines $P$ w.r.t the clock semantic and the dependence. This will provide formal guarantee as strong as that provided by a formally certified compiler. Indeed, consider the following process:

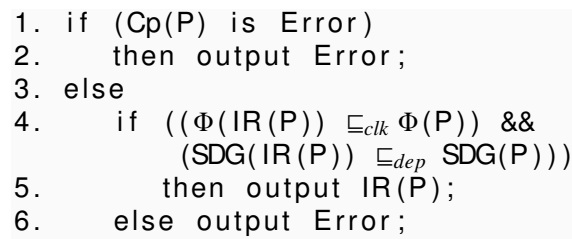

We describe the main components of the implementation which is integrated in the existing Polychrony toolset [32] to prove the preservation of clock semantics and dependence of the Signal compiler. We are interested in the two first stages: clock calculation, boolean abstraction and static scheduling. The intermediate forms in the transformations of the compiler may be expressed in the Signal language itself.

At a high level, our tool which is depicted in Figure 4 works as follows. First, it takes the input program P.SIG and its transformed program $\mathrm{P}_{-}$TRA.SIG, computes the corresponding clock models. The clock models of input and transformed programs are combined as the formula $\left(\Phi\left(P \_T R A . S I G\right) \Rightarrow \Phi(P . S I G)\right)$. It uses a solver to check

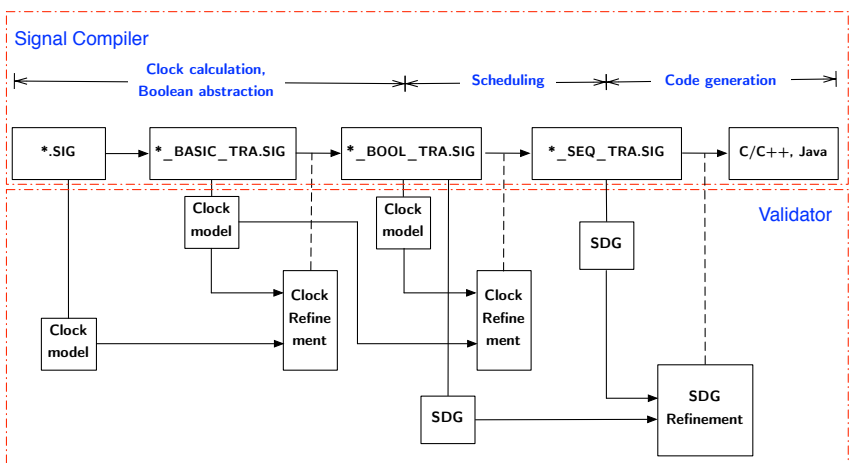

Fig. 4 An overview of our integration within Polychrony toolset

$\vDash(\Phi($ P_TRA.SIG $) \Rightarrow \Phi(\mathrm{P} . \mathrm{SIG}))$ (or equivalently $M \not$ $\neg\left(\Phi\left(P_{-}\right.\right.$TRA.SIG $) \Rightarrow \Phi($ P.SIG $\left.)\right)$. The result of this checking can be exploited for the preservation of clock semantic of the transformations. If the result says that the checked formula is not valid (or the negation formula is satisfiable) then it emits compilation error. Otherwise, the compiler continues its work. The same procedure is applied for the other steps of the compiler. Finally, our verification process asserts that $\Phi\left(\mathrm{P} \_\right.$BOOL_TRA.SIG $) \sqsubseteq_{c l k} \Phi\left(\mathrm{P} \_\right.$TRA.SIG $) \sqsubseteq_{c l k} \Phi(\mathrm{P} . \mathrm{SIG})$ along the transformations of the compiler.

In the similar way, for the scheduling stage of the compilation, our tool takes the program $\mathrm{P}_{-} \mathrm{BOOL}$ TRA.SIG and its transformed program P_SEQ_TRA.SIG, constructs the corresponding SDGs. Then it checks that SDG(P_SEQ_

TRA.SIG) is a dependence refinement of SDG(P_BOOL_TRA.

$\mathrm{SIG})$. If the answer is "No", then it emits compilation error. Otherwise, the compiler continues its work.

Here, we delegate the checking of the refinements to a SMT solver. Our implementation uses the SMT-LIB common format [11] to encode the clock models as input of the SMTsolver. For our implementation, we consider the Yices [14] solver, which is one of the best solvers at the SMT-COMP competition [37].

\subsection{Constant Clock and Renaming}

In Signal, the occurrence of constants is allowed to designate a constant signal (e.g. a signal with a constant value). However, each occurrence of a constant has a particular clock since the corresponding signal is hidden, this clock is determined by the context where the constant is used, called context clocks. This makes our abstraction for Signal operator above invalid in case a constant signal is used. In consequence of that, we provide the abstraction for each Signal op- 
erator when this operator uses a constant signal, where cst denotes a constant.

\subsubsection{Stepwise Extensions}

- $\phi(y:=c s t)=\hat{y} \Rightarrow(\bar{y} \Leftrightarrow c s t)$ if $y$ is Boolean.

- $\phi(y:=c s t)=\emptyset$ if $y$ is non-Boolean.

- $\phi(y:=x$ and $c s t)=(\hat{y} \Leftrightarrow \hat{x}) \wedge(\hat{y} \Rightarrow(\bar{y} \Leftrightarrow \bar{x} \wedge c s t))$.

- $\phi(y:=x$ or cst $)=(\hat{y} \Leftrightarrow \hat{x}) \wedge(\hat{y} \Rightarrow(\bar{y} \Leftrightarrow \bar{x} \vee c s t))$.

- $\phi(y:=x \square c s t)=\left(\hat{y} \Leftrightarrow \widehat{v_{\square}^{i}} \Leftrightarrow \hat{x}\right) \wedge\left(\hat{y} \Rightarrow\left(\bar{y}=\overline{v_{\square}^{i}}\right)\right)$.

\subsubsection{Deterministic Merge}

- $x$ and $y$ are Boolean.

$\phi(y:=x$ default $c s t)=(\hat{y} \Leftrightarrow(\hat{x} \vee \hat{y})) \wedge \hat{y} \Rightarrow((\hat{x} \wedge(\bar{y} \Leftrightarrow$

$\bar{x})) \vee(\neg \hat{x} \wedge(\bar{y} \Leftrightarrow c s t))))$

$\phi(y:=$ cst default $x)=(\hat{y} \Leftrightarrow(\hat{x} \vee \hat{y})) \wedge \hat{y} \Rightarrow((\hat{y} \wedge(\bar{y} \Leftrightarrow$ $c s t)) \vee(\neg \hat{y} \wedge(\bar{y} \Leftrightarrow \bar{x}))))$

- $x, y$ and $z$ are non-Boolean.

$\phi(y:=x$ default $c s t)=\hat{y} \Leftrightarrow(\hat{x} \vee \hat{y})$

$\phi(y:=c s t$ default $x)=\hat{y} \Leftrightarrow(\hat{x} \vee \hat{y})$

\subsubsection{Boolean Sampling}

- $x$ and $y$ are Boolean.

$\phi(y:=x$ when true $)=(\hat{y} \Leftrightarrow(\hat{x} \wedge \hat{y})) \wedge(\hat{y} \Rightarrow(\bar{y} \Leftrightarrow \bar{x}))$

$\phi(y:=x$ when false $)=\hat{y} \Leftrightarrow$ false

$\phi(y:=c s t$ when $b)=(\hat{y} \Leftrightarrow(\hat{b} \wedge \bar{b})) \wedge(\hat{y} \Rightarrow(\bar{y} \Leftrightarrow c s t))$

- $x$ and $y$ are non-Boolean.

$\phi(y:=x$ when true $)=\hat{y} \Leftrightarrow(\hat{x} \wedge \hat{y})$

$\phi(y:=x$ when false $)=\hat{y} \Leftrightarrow$ false

$\phi(y:=c s t$ when $b)=\hat{y} \Leftrightarrow(\hat{b} \wedge \bar{b})$

Consider a process $P$ and its sub-process $P_{1}$ such that a signal named $\mathrm{x}$ is local variable of both $P$ and $P_{1}$. When compiling this program, the compiler rename variable $\mathrm{x}$ in the sub-process $P_{1}$. Our validator requires that the mapping of the original name and the new name for every variable such as $x$. Based on this mapping, for every variable $x$ and its new name $x_{-} i$, the following conjunct is added to the clock model:

- $\left(\hat{x} \Leftrightarrow \widehat{x_{-} i}\right) \wedge\left(\bar{x} \Leftrightarrow \overline{x_{-} i}\right)$ if $x$ is Boolean.

- $\left(\hat{x} \Leftrightarrow \widehat{x_{-} i}\right) \wedge\left(\bar{x}=\overline{x_{-} i}\right)$ if $x$ is non-Boolean.

\subsection{Detected Bugs}

So far out validator has revealed 2 previously-unknown bugs in the compilation of the Signal compiler, one of them is related to the multiple constraints of clock. Another is a syntax error of generated $\mathrm{C}$ code from a Signal program in which a constant signal is presented.

The first problem was introduced when multiple constraints condition a clock such as the following segment of Signal program and its clock calculation parts in transformed programs

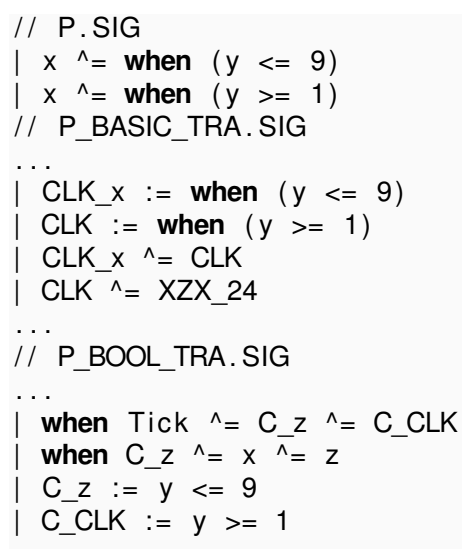

In the transformed counterpart $P_{-}$BASIC_TRA, the introduction of signal XZX_24 and the synchronization between CLK and XZX_24 cause the incorrect specification of clocks (e.g. in the source program $\mathrm{P}$ and $\mathrm{P} \_\mathrm{BOOL}$ TRA, signal $\mathrm{x}$ is present, but in program P_BASIC_TRA, it might be absent when XZX_24 is absent). This bug be caught by our validator when it found that $\Phi\left(\mathrm{P} \_\right.$BOOL_TRA $) \nsubseteq_{c l k} \Phi($ P_BASIC_TRA). In addition, signal XZX_24 is introduced without declaration that makes a syntax error in P_BASIC_TRA.

The second problem was present in the Signal program in which a merge operator with a constant signal is used such as $y:=1$ default $x$. The clock calculation is correct based on the validator result when it check the clock refinements between the input and transformed counterparts. However, it seems that the code generation phase of the compiler deals wrongly with the clock context of a constant signal by introducing a syntax error in the generated $\mathrm{C}$ code. The bug and its fix are given by:

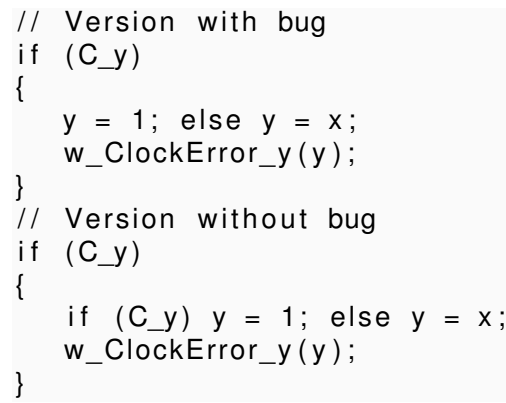




\section{Conclusions and Related Work}

The notion of translation validation was introduced in [33,34] by A. Pnueli et al. to verify the code generator of Signal. In that work, the authors define a language of symbolic models to represent both the source and target programs, called Synchronous Transition Systems (STS). A STS is a set of logic formulas which describe the functional and temporal constraints of the whole program and its generated $\mathrm{C}$ code. Then they use BDD [35] representations to implement the symbolic STS models, and their proof method uses a SAT-solver to reason on the signal constraints. The drawback of this approach is that it does not capture explicitly the clock semantic and data dependences and in some cases, the compiler eliminates the use of a local register variable in the generated code and then, the mapping cannot be established. Additionally, for a large program, the formula is very large, including numerical expressions that make some inefficiency. Moreover, the whole calculation of a synchronous program or the generated code is considered as one atomic transition in STS, thus it does not capture the scheduling semantics, data dependencies of the programs and does not explicitly prove the preservation of abstract clocks and data dependences. Another related work is the static analysis of Signal programs for efficient code generation [19]. In a similar way, they formalize the abstract clocks and clock relations as first-order logic formulas with the help of interval abstraction technique. Then, to make the generated code more efficient by detecting and removing the dead-code segments (e.g., segment of code for the signals whose clocks are always empty). The approach is that they determine the existence of empty clocks, mutual exclusion of two or more clocks, or clock inclusion by reasoning on the formal model using a SMT-solver. There have been some other works which adopt the translation validation approach in verification of transformations, and optimizations. In $[12,28]$, the programs before and after the transformations and optimizations of $\mathrm{C}$ compiler are represented in a common intermediate form, then the preservation of semantics is checked by using symbolic execution and the proof assistant Coq [13]. With the same purpose, in the work of [30], the source programs and its transformed counterpart are encoded with Polynomial Dynamical Systems. By using the simulation in model checking techniques to prove the preservation of clock semantic, this approach suffers from the increasing of the state-space when it deals with large programs. On the contrary, in our present work, the clock constraints are de- scribed as a logic formula over Boolean variables. With an efficient SMT-solver in processing these formulas, our approach can deal with large programs that make the state-space explosion problem in model checking techniques.

The present paper provides a proof of preservations of clock semantic and the dependence between signals during the transformations of the Signal compiler. We have presented a technique based on SMT-solving to check the existence of these preservations. The desired behavior of a given source program and its transformed counterpart are represented as clock models and SDGs. Refinement relations between clock models and synchronous data-flow graphs are used to express the preservations, which are checked by using a SMT-solver.

We have implemented and integrated our translation validation process within the Polychrony toolset by using the Yices solver to prove the correctness of the full compilation phases of the compiler. As future work, first, we would like to extend our work to the final phase of the Signal compiler, the code generation. That means the data dependence between variables in the sequential generated code $C$ will be represented as a SDG, then the rest of the verification process is the same as the present work. Second, we would like to use the proof of abstract clock semantic preservation in this work to verify the equivalence between data-flows and the corresponding variables from the program and its generated code. The verification of equivalence will be done by using a normalizing value-graph [39] which contains only the computations of data-flows and there is no timing information. We therefore evaluate this graph more efficiently.

Acknowledgements We would like to thank Sandeep Shukla for his early interest and enthusiasm, and Abdoulaye Gamatié, Laure Gonnord for discussing some parts of this work and exchanging ideas.

\section{References}

1. W. Ackerman. Solvable cases of the Decision Problem. Study in Logic and the Foundations of Mathematics. North-Holland, Amsterdam, 1954.

2. W.B. Ackerman. Data flow languages. Computer. vol. 15, Feb 1982.

3. S. Abramsky and A. Jung: Domain theory: Handbook of logic in computer science. Clarendon Press. pp.1-168, 1994.

4. F.E. Allen. Control flow analysis. In proceedings of a Symposium on Compiler Optimization, SIGPLAN Not. 5, 7. pp1-19, Jul 1970.

5. A.V. Aho, R. Sethi, and J.D. Ullman. Compilers: Principles, Techniques, and Tools (Second edition). Addison-Wesley Reading, MA, 2007 
6. A. Benveniste, P. Caspi, S.A. Edwards, N. Halbwachs, P. Le Guernic, and R. De Simone. The synchronous languages 12 years later. Proceedings of the IEEE 91(1). pp.64-83, 2003.

7. G. Berry: The foundations of Esterel. In Proof, Language and Interaction: Essay in Honor of Robin Milner, MIT Press, 2000

8. L. Besnard, T. Gautier, P. Le Guernic, and J-P. Talpin. Compilation of polychronous data flow equations. In Synthesis of Embedded Software, Springer, 2010.

9. A. Benveniste and P. Le Guernic. Hybrid dynamical systems theory and the Signal language. IEEE Transactions on Automatic Control, 35(5):535-546, May 1990.

10. A. Biere, M. Heule, H. van Maaren, and T. Walsh. Handbook of satisfiability: Volume 185 Frontiers in Artificial Intelligence and Applications. IOS Press, Amsterdam, The Netherlands. ISBN 978-1-58603929-5, 2009.

11. C. Barrett, S. Ranise, A. Stump, and C. Tinelli. The Satisfiability Modulo Theories Library (SMT-LIB). http://www.SMT-LIB.org, 2008.

12. Inria. The CompCert Project. http://compcert.inria.fr.

13. Inria. The Coq Proof Assitant. http://coq.inria.fr.

14. B. Dutertre, and L. de Moura. Yices sat-solver. http://yices.csl.ri.com, 2009.

15. J. Ferrante, K.J. Ottenstein, and J.D. Warren. The program dependence graph and its use in optimization. ACM Transactions on Programming Languages and Systems, 9(3):319-349, Jul 1987.

16. A. Gamatié. Designing embedded systems with the Signal programming: Synchronous, Reactive specification. Springer, New York. ISBN 978-1-4419-0940-4, 2009

17. J.H. Gallier. Logic for computer science. John Wiley. 1987.

18. G.G. De Jong. Generalized data-flow graphs: Theory and applications. Ph.D thesis, Eindhoven University of Technology, 1993.

19. A. Gamatié, and L. Gonnord. Static Analysis of Synchronous Programs in Signal for Efficient Design of Multi-Clocked Embedded Systems. In ACM SIGPLAN/SIGBED Conference on Languages, Compilers, Tools and Theory for Embedded Systems - LCTES'2011. Chicago, IL, USA, April 2011.

20. P. Le Guernic, and T. Gautier. Advanced topics in data-flow computing, chapter data-flow to von Neumann: the Signal approach. PrenticeHall. pp.413-438, 1991.

21. T. Gautier, P. Le Guernic, and L. Besnard. Signal, a declarative language for synchronous programming of real-time systems. In Proc. 3rd. Conf. on Functional Programming Languages and Computer Architecture. LNCS 274, Springer Verlag, May 1990.

22. P. Le Guernic, J-P. Talpin, and J-C. Le Lann. Polychrony for system design. Journal for Circuits, Systems and Computers. 12(3):261-304, Apr 2003.
23. N. Halbwachs. A synchronous language at work: the story of Lustre. In 3th ACM-IEEE International Conference on Formal Methods and Models for Codesign (MEMOCODE'05), Jul 2005.

24. M. Huth, and M. Ryan. Logic in computer science: Modelling and Reasoning about systems. Cambridge University Press. ISBN 978-05215-4310-1, 2004.

25. G. Kahn: The Semantics of simple language for parallel programming IFIP Congress. pp.471-475, 1974.

26. P. Le Guernic, T. Gautier, M. Le Borgne, and C. Le Maire. Programming real-time applications with Signal. In Proceedings of the IEEE, 79(9):1321-1336, Sep 1991.

27. L. de Moura, and N. Bjorner. Satisfiability Modulo Theories: An appetizer. In Brazilian Symposium on Formal Methods (SBMF'2009), Gramado, Brazil, Aug 2009.

28. G.C. Necula. Translation Validation for an Optimizing Compiler. In Proceeding PLDI'OO Proceedings of the ACM SIGPLAN 2000 Conference on Programming Language Design and Implementation. pp.8394, May 2000.

29. O. Maffeis, and P. LeGuernic. Combining ependability with architectural adaptability by means of the signal language. Static Analysis, LNCS 724, 1993

30. V.C. Ngo, J-P. Talpin, T. Gautier, P. Le Guernic, and L. Besnard. Formal Verification of Compiler Transformations on Polychronous Equations. In Proceedings of IFM'12, LNCS 7321. pp.113-127, 2012.

31. V.C. Ngo, J-P. Talpin, T. Gautier, and P. Le Guernic. Formal Verification of Transformations on Abstract Clocks in Synchronous Compilers. In HAL-INRIA, Technical Report RR-8064, Sep 2012.

32. Espresso, Polychrony Toolset. http://www.irisa.fr/espresso/Polychrony.

33. A. Pnueli, M. Siegel, and E. Singerman. Translation validation. In B. Steffen, editor, 4th Intl. Conf. TACAS'98. LNCS 1384. pp.151-166, 1998.

34. A. Pnueli, O. Shtrichman, and M. Siegel. Translation validation: From Signal to C. In Correct Sytem Design Recent Insights and Advances. LNCS 1710. pp.231-255, 2000.

35. R. Bryant: Graph-based algorithms for boolean function manipulation. IEEE Transactions on Computers, C-35(8):677-691, Aug 1986.

36. DO-178C. http://rtca.org

37. A. Stump, and M. Deters. http://www.smtcomp.org/2009, 2009.

38. S.P. Rajan. Transformations on Dependency Graphs: Formal Specification and Efficient Mechanical Verification. Ph.D thesis, University of British Columbia, Vancouver, Canada.

39. J-B. Tristan, P. Govereau, and G. Morrisett. Evaluating value-graph translation validation for LLVM. In ACM SIGPLAN Conference on Programming and Language Design Implementation. California, 2011. 